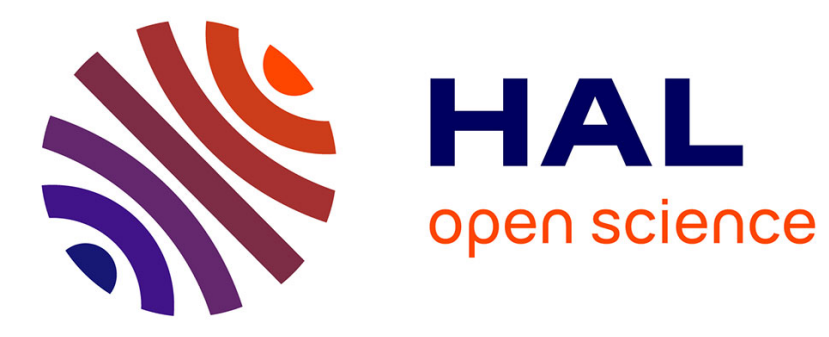

\title{
Laguerre and Hermite bases for inverse problems
}

Fabienne Comte, Valentine Genon-Catalot

\section{To cite this version:}

Fabienne Comte, Valentine Genon-Catalot. Laguerre and Hermite bases for inverse problems . Journal of the Korean Statistical Society, 2018, 47 (3), pp.273-296. 10.1016/j.jkss.2018.03.001 . hal$01449799 \mathrm{v} 2$

\section{HAL Id: hal-01449799 \\ https://hal.science/hal-01449799v2}

Submitted on 4 Oct 2017

HAL is a multi-disciplinary open access archive for the deposit and dissemination of scientific research documents, whether they are published or not. The documents may come from teaching and research institutions in France or abroad, or from public or private research centers.
L'archive ouverte pluridisciplinaire HAL, est destinée au dépôt et à la diffusion de documents scientifiques de niveau recherche, publiés ou non, émanant des établissements d'enseignement et de recherche français ou étrangers, des laboratoires publics ou privés. 


\title{
LAGUERRE AND HERMITE BASES FOR INVERSE PROBLEMS
}

\author{
F. COMTE $^{(1)} \&$ V. GENON-CATALOT ${ }^{(2)}$
}

\begin{abstract}
We present inverse problems of nonparametric statistics which have a performing and smart solution using projection estimators on bases of functions with non compact support, namely, a Laguerre basis or a Hermite basis. The models are $Y_{i}=X_{i} U_{i}, Z_{i}=X_{i}+\Sigma_{i}$, where the $X_{i}$ 's are i.i.d. with unknown density $f$, the $\Sigma_{i}$ 's are i.i.d. with known density $f_{\Sigma}$, the $U_{i}$ 's are $i . i . d$. with uniform density on $[0,1]$. The sequences $\left(X_{i}\right),\left(U_{i}\right),\left(\Sigma_{i}\right)$ are independent. We define projection estimators of $f$ in the two cases of indirect observations of $\left(X_{1}, \ldots, X_{n}\right)$, and we give upper bounds for their $\mathbb{L}^{2}$-risks on specific Sobolev-Laguerre or Sobolev-Hermite spaces. Data-driven procedures are described and proved to perform automatically the bias variance compromise.
\end{abstract}

(1) Université Paris Descartes, MAP5, UMR CNRS 8145, email: fabienne.comte@parisdescartes.fr

(2) Université Paris Descartes, MAP5, UMR CNRS 8145, valentine.genon-catalot@parisdescartes.fr.

Keywords. Adaptive estimation. Additive noise. Inverse problem. Model selection. Multiplicative censoring. Projection estimator.

MSC2010. 62G05 - 62G07

\section{INTRODUCTION}

The aim of this paper is to demonstrate that some classical inverse problems of nonparametric statistics can be dealt with by using projection estimators either on a Laguerre basis or on a Hermite basis and that this approach provides easy and performing solutions. We make a unified presentation joining novelties and improvements of previous results.

To motivate the framework, first consider $X_{1}, \ldots, X_{n} n$ i.i.d. random variables with unknown density $f$. If the $X_{i}$ 's are observed and $f$ is square integrable, nonparametric estimators of $f$ can be built by using a projection method on an orthonormal basis. Many authors use compactly supported bases (see e.g. Massart (2007), Efromovich (1999)). Non compactly supported wavelet bases of $\mathbb{L}^{2}(\mathbb{R})$ have also been used (see Juditsky and Lambert-Lacroix (2004), and the references therein). The $\mathbb{L}^{2}(\mathbb{R})$ basis of Hermite functions is another possibility, investigated in e.g. Schwarz (1967), Walter (1977) and more recently, in Belomestny et al., 2017. As enlighted in the latter paper, projection estimators on a Hermite basis have the following advantages. First, as the basis is not compactly supported, there is no need to fix an interval for estimation. This is convenient, especially for inverse problems. Second, from the computational point of view, they have a much lower complexity than other estimators based on non compactly supported bases such as deconvolution estimators. Nevertheless they perform asymptotically as well as estimators built from competing methods. If $f$ is $\mathbb{R}^{+}$-supported, one can use an orthonormal basis of $\mathbb{L}^{2}\left(\mathbb{R}^{+}\right)$. The Laguerre basis is then well fitted. The qualities of Laguerre projection estimators have been recently investigated (see e.g. Mabon (2017), Vareschi (2015), and for actuarial applications Zhang and $\mathrm{Su}(2017)$ ). If the $X_{i}$ 's are not directly observed, the estimation of $f$ is an inverse problem. The inverse problems considered here are the following ones. First,

Date: October 4, 2017. 
we assume that observations are

$$
Y_{i}=X_{i} U_{i}, \quad i=1, \ldots, n
$$

where the sequences $\left(X_{i}\right),\left(U_{i}\right)$ are independent and $\left(U_{i}\right)$ are i.i.d. with uniform distribution on $[0,1]$. Model (1) is called multiplicative censoring model and covers several important statistical problems (see e.g. Vardi (1989)). Numerous papers deal with the estimation of $f$ for model (1) whether by nonparametric maximum likelihood (Vardi (1989), Vardi and Zhang (1992), Asgharian et al. (2012)), by projection methods (Andersen and Hansen (2001), Abbaszadeh et al. $(2012,2013)$ ) or kernel methods (Brunel et al. (2015)). In Belomestny et al. (2016), $f$ is supposed to be $\mathbb{R}^{+}$-supported and estimated by projection estimators on a Laguerre basis. We revisit this problem and obtain an improvement of the risk bound, under a slight additional moment assumption. In the case of real-valued random variables $X_{i}$, we investigate the estimation of $f$ from model (1) by using a Hermite basis approach.

Second, we consider observations $Z_{1}, \ldots, Z_{n}$ such that

$$
Z_{i}=X_{i}+\Sigma_{i}, \quad i=1, \ldots, n .
$$

where $\left(\Sigma_{i}\right)$ are i.i.d. with known density $f_{\Sigma}$ and the sequences $\left(X_{i}\right),\left(\Sigma_{i}\right)$ are independent. Density estimation from noisy observations is the subject of a huge number of contributions. This deconvolution problem is classically solved by Fourier methods (see e.g. Comte et al. (2006) and the references therein). Recently, when the $X_{i}$ 's are non-negative, the study of one-sided errors, i.e. $\Sigma_{i} \geq 0$, was motivated by applications in the field of finance (see Jirak et al. (2014)) or in survival models, (see van Es et al. (1998), Jongbloed (1998)). In particular, Mabon (2017) proposes for model (2) projection estimators of $f$ using a Laguerre basis whose properties allow deconvolution of densities on $\mathbb{R}^{+}$. We detail this approach and provide an improvement of the risk bound of the projection estimators. In the case of real-valued random variables $X_{i}, \Sigma_{i}$, we study the estimation of $f$ by using a Hermite basis approach.

To our knowledge, the estimation of $f$ from observations (1)-(2) by projection estimators on a Hermite basis, which is investigated here, is new. We compare Hermite estimators to the deconvolution estimators for models (1) and (2). Note that the estimation of $f$ in model (1) by deconvolution is also new.

In each of the above models, we exhibit explicit relations between the projection coefficients of the density of the observed variables and the projection coefficients of the unknown density $f$. This allows to build projection estimators of $f$. We provide risk bounds for the estimators. Afterwards, we propose data-driven procedures, including for model (1) a random penalty which avoids a priori knowledge of the variance rate. This is important as the variance order varies in function of moment assumptions.

Laguerre and Hermite bases are related to specific function spaces, respectively the SobolevLaguerre spaces (see e.g. Shen (2000) and Bongioanni and Torrea (2007)) or the Sobolev-Hermite spaces (Bongioanni and Torrea (2006)). The links between rate of decay of the projection coefficients and regularity properties of functions in these spaces have been described respectively in Comte and Genon-Catalot (2015) and in Belomestny et al. (2017). This allows to assess the rate of bias terms of $\mathbb{L}^{2}$-risks in the projection approach, and to compute upper bounds for the rates of convergence.

In Section 2, we describe the Laguerre and Hermite bases and spaces. In Section 3, for the purpose of comparison with the other models, we study the case of direct observations of $X_{1}, \ldots, X_{n}$. Section 4 deals with model (1). Section 5 is concerned with model (2). In Section 6 , we give some concluding remarks. Section 7 contains useful formulae for Laguerre and Hermite 
functions and all proofs. In the Appendix, we recall some properties of Sobolev-Laguerre and Sobolev-Hermite spaces, and the Talagrand inequality used in proofs.

\section{About Laguerre and Hermite bases and spaces}

2.1. Laguerre and Hermite bases. We start by presenting the Laguerre and Hermite bases and the Sobolev-Laguerre and Sobolev-Hermite regularity spaces. More details on Laguerre and Hermite functions are given in Section 7.1.

Below we denote the scalar product and the $\mathbb{L}^{2}$-norm on $\mathbb{L}^{2}\left(\mathbb{R}^{+}\right)$by: $\forall s, t \in \mathbb{L}^{2}\left(\mathbb{R}^{+}\right),\langle s, t\rangle_{+}=$ $\int_{0}^{+\infty} s(x) t(x) d x,\|t\|_{+}^{2}=\int_{0}^{+\infty} t^{2}(x) d x$. We also denote by $\|\cdot\|$ the $\mathbb{L}^{2}$-norm on $\mathbb{R}$ and by $\langle\cdot, \cdot\rangle$ the $\mathbb{L}^{2}(\mathbb{R})$-scalar product.

Consider the Laguerre polynomials $\left(L_{j}\right)$ and the Laguerre functions $\left(\varphi_{j}\right)$ given by

$$
L_{j}(x)=\sum_{k=0}^{j}(-1)^{k}\left(\begin{array}{l}
j \\
k
\end{array}\right) \frac{x^{k}}{k !}, \quad \varphi_{j}(x)=\sqrt{2} L_{j}(2 x) e^{-x} \mathbf{I}_{x \geq 0}, \quad j \geq 0 .
$$

The collection $\left(\varphi_{j}\right)_{j \geq 0}$ constitutes a complete orthonormal system on $\mathbb{L}^{2}\left(\mathbb{R}^{+}\right)$, and is such that (see Abramowitz and Stegun (1964)):

$$
\forall j \geq 0, \quad \forall x \in \mathbb{R}^{+}, \quad\left|\varphi_{j}(x)\right| \leq \sqrt{2} .
$$

For $f \in \mathbb{L}^{2}\left(\mathbb{R}^{+}\right)$, we can develop $f$ on the Laguerre basis, $f=\sum_{j \geq 0} a_{j}(f) \varphi_{j}, \quad a_{j}(f)=\left\langle f, \varphi_{j}\right\rangle_{+}$. The Hermite polynomial and the Hermite function of order $j$ are given, for $j \geq 0$, by:

$$
H_{j}(x)=(-1)^{j} e^{x^{2}} \frac{d^{j}}{d x^{j}}\left(e^{-x^{2}}\right), \quad h_{j}(x)=c_{j} H_{j}(x) e^{-x^{2} / 2}, \quad c_{j}=\left(2^{j} j ! \sqrt{\pi}\right)^{-1 / 2}
$$

The sequence $\left(h_{j}, j \geq 0\right)$ is an orthonormal basis of $\mathbb{L}^{2}(\mathbb{R})$. When the density $f$ belongs to $\mathbb{L}^{2}(\mathbb{R})$, it can be developed in the Hermite basis $f=\sum_{j \geq 0} a_{j}(f) h_{j}$ where $a_{j}(f)=\int_{\mathbb{R}} f(x) h_{j}(x) d x=$ $\left\langle f, h_{j}\right\rangle$. The infinite norm of $h_{j}$ satisfies (see Abramowitz and Stegun (1964), Szegö (1959) p.242):

$$
\left\|h_{j}\right\|_{\infty} \leq \Phi_{0}, \quad \Phi_{0} \simeq 1,086435 / \pi^{1 / 4} \simeq 0.8160,
$$

and the following more precise bound is available (with $C_{\infty}$ a constant given in Szegö (1959))

$$
\left\|h_{j}\right\|_{\infty} \leq \frac{C_{\infty}}{(j+1)^{1 / 12}}, \quad j=0,1, \ldots
$$

We use the notation $\psi_{j}$ to designate $\varphi_{j}$ in the Laguerre case and $h_{j}$ in the Hermite case, denote by $S_{m}=\operatorname{span}\left(\psi_{0}, \psi_{1}, \ldots, \psi_{m-1}\right)$ the linear space generated by the $m$ functions $\psi_{0}, \ldots, \psi_{m-1}$ and by $f_{m}=\sum_{j=0}^{m-1} a_{j}(f) \psi_{j}$ the orthogonal projection of $f$ on $S_{m}$, where $a_{j}(f)=\left\langle f, \psi_{j}\right\rangle$ will mean either $\left\langle f, \varphi_{j}\right\rangle_{+}$or $\left\langle f, h_{j}\right\rangle$.

2.2. Sobolev-Laguerre and Sobolev-Hermite spaces. For $s \geq 0$, the Sobolev-Laguerre space with index $s$ (see Bongioanni and Torrea (2007)) is defined by:

$$
W_{L}^{s}=\left\{\theta: \mathbb{R}^{+} \rightarrow \mathbb{R}, \theta \in \mathbb{L}^{2}\left(\mathbb{R}^{+}\right),|\theta|_{s}^{2}:=\sum_{k \geq 0} k^{s} a_{k}^{2}(\theta)<+\infty\right\}
$$

where $a_{k}(\theta)=\left\langle\theta, \varphi_{k}\right\rangle_{+}$. We define the ball $W_{L}^{s}(D)$ by

$$
W_{L}^{s}(D) \doteq\left\{\theta \in W_{L}^{s},|\theta|_{s}^{2}=\sum_{k=0}^{\infty} k^{s} a_{k}^{2}(\theta) \leq D\right\} .
$$


Analogously, the Sobolev-Hermite space with regularity $s$ (see Bongioanni and Torrea (2006)) is given by

$$
W_{H}^{s}=\left\{\theta: \mathbb{R} \rightarrow \mathbb{R}, \theta \in \mathbb{L}^{2}(\mathbb{R}), \sum_{k \geq 0} k^{s} a_{k}^{2}(\theta)<+\infty\right\} .
$$

where $a_{k}(\theta)=\left\langle\theta, h_{k}\right\rangle$, and the Sobolev-Hermite ball

$$
W_{H}^{s}(D)=\left\{\theta \in \mathbb{L}^{2}(\mathbb{R}), \sum_{k \geq 0} k^{s} a_{k}^{2}(\theta) \leq D\right\} .
$$

Thus, for $f$ in $W_{L}^{s}(D)$ or in $W_{H}^{s}(D)$, we have $\left\|f-f_{m}\right\|^{2} \leq D m^{-s}$.

For details and especially for regularity properties of functions in these spaces, we refer also to Section 7 of Comte and Genon-Catalot (2015), Section 7.2 of Belomestny et al. (2016) and Section 4.1 of Belomestny et al. (2017), see Appendix A.

\section{Projection estimators of $f$ When $X_{i}$ 's are observed}

3.1. Risk bound. We assume that $f$ belongs to $\mathbb{L}^{2}\left(\mathbb{R}^{+}\right)$or $\mathbb{L}^{2}(\mathbb{R})$ and provide for each $m \geq 1$, a projection estimator of $f$ by estimating the coefficients $a_{j}(f), j=0, \ldots, m-1$. In the case where the $X_{i}$ 's are observed, we define the empirical and unbiased estimator of $a_{j}(f)$ by

$$
\hat{a}_{j}(X)=\frac{1}{n} \sum_{i=1}^{n} \psi_{j}\left(X_{i}\right) \text { and the projection estimator } \hat{f}_{m}^{X}=\sum_{j=0}^{m-1} \hat{a}_{j}(X) \psi_{j} .
$$

Clearly, $\hat{f}_{m}^{X}$ an unbiased estimator of $f_{m}=\sum_{j=0}^{m-1} a_{j}(f) \psi_{j}$, the orthogonal projection of $f$ on $S_{m}$. By the Pythagoras Theorem, we have $\left\|\hat{f}_{m}^{X}-f\right\|^{2}=\left\|f-f_{m}\right\|^{2}+\left\|\hat{f}_{m}^{X}-f_{m}\right\|^{2}$. As $\left(\psi_{j}\right)_{j}$ is orthonormal, we get $\left\|\hat{f}_{m}^{X}-f_{m}\right\|^{2}=\sum_{j=0}^{m-1}\left(\hat{a}_{j}(X)-a_{j}(f)\right)^{2}$ and

$$
\mathbb{E}\left[\left(\hat{a}_{j}(X)-a_{j}(f)\right)^{2}\right]=\frac{1}{n} \operatorname{Var}\left(\psi_{j}\left(X_{1}\right)\right)=\frac{1}{n} \mathbb{E}\left(\psi_{j}^{2}\left(X_{1}\right)\right)-\frac{a_{j}^{2}(f)}{n} .
$$

Let us define

$$
V_{m}^{X}=\sum_{j=0}^{m-1} \mathbb{E}\left[\psi_{j}^{2}\left(X_{1}\right)\right]
$$

we have

$$
\mathbb{E}\left(\left\|\hat{f}_{m}^{X}-f\right\|^{2}\right)=\left\|f-f_{m}\right\|^{2}+\frac{V_{m}^{X}}{n}-\frac{\left\|f_{m}\right\|^{2}}{n} .
$$

With (4) or (6), we obtain $V_{m}^{X} \leq C m$, with $C=2$ in the Laguerre case and $C=\Phi_{0}^{2}$ (see (6)) in the Hermite case. In the latter case, using (7), we get $V_{m}^{X} \leq C m^{5 / 6}$, which is better. However, these bounds on the variance term are not optimal and can be improved under rather weak moment assumptions.

Proposition 3.1. If $\mathbb{E}\left(1 / \sqrt{X_{1}}\right)<+\infty$ in the Laguerre case or $\mathbb{E}\left(\left|X_{1}\right|^{2 / 3}\right)<+\infty$ in the Hermite case, then for $m$ large enough, $V_{m}^{X} \leq c \sqrt{m} / n$ where $c$ is a constant, and thus

$$
\mathbb{E}\left(\left\|\hat{f}_{m}^{X}-f\right\|^{2}\right) \leq\left\|f-f_{m}\right\|^{2}+c \frac{\sqrt{m}}{n} .
$$


In the Laguerre case, this result improves the variance order obtained in Belomestny et al. (2016), under the additional moment assumption $\mathbb{E}\left(1 / \sqrt{X_{1}}\right)<+\infty$. For the Hermite case, the order $O(\sqrt{m})$ is obtained in Belomestny et al. (2017b), Proposition 2.1, but under the stronger moment condition $\mathbb{E}\left(\left|X_{1}\right|^{5}\right)<+\infty$. Note that even the Cauchy distribution satisfies $\mathbb{E}\left(\left|X_{1}\right|^{2 / 3}\right)<+\infty$.

The risk bound decomposition (12) involves a bias term $\left\|f-f_{m}\right\|^{2}=\sum_{j \geq m} a_{j}^{2}(f)$ which is decreasing with $m$ and a variance term of order $\sqrt{m} / n$ which is increasing with $m$. Therefore, to evaluate the rate of convergence, we have to perform a compromise to select relevantly $m$. More precisely, for $f$ in $W_{L}^{s}(D)$ or in $W_{H}^{s}(D)$, we have $\left\|f-f_{m}\right\|^{2}=\sum_{j \geq m} a_{j}^{2}(f) \leq D m^{-s}$. Choosing $m_{\mathrm{opt}}=\left[n^{1 /(s+1 / 2)}\right]$ in the r.h.s. of (12) implies

$$
\mathbb{E}\left(\left\|\hat{f}_{m_{\mathrm{opt}}}^{X}-f\right\|^{2}\right) \leq C_{0}(s, D) n^{-2 s /(2 s+1)}
$$

where $C_{0}(s, D)$, is a constant depending on $s$ and $D$ only. This is the usual rate for density estimation for $f$ belonging to a classical Sobolev ball with regularity $s$ : indeed, Schipper (1996) proves that this rate is minimax optimal with exact Pinsker constant on Sobolev balls for an integer $s$, Efromovich (2002) proves the result for $s<1 / 2$ and Rigollet (2006) builds an adaptive deconvolution estimator based on the blockwise Stein method, which reaches the optimal rate with exact constant for any $s>1 / 2$.

The upper bound $O(\sqrt{m})$ on the term $V_{m}^{X}$ is somehow optimal as we can prove:

Proposition 3.2. Assume that $\inf _{a \leq x \leq b} f(x)>0$ for some interval $[a, b]$, with $a<b$ in the Hermite case and $0<a<b$ in the Laguerre case, then, for $m$ large enough, $V_{m}^{X} \geq c^{\prime} \sqrt{m}$ where $c^{\prime}$ is a constant. Therefore

$$
\mathbb{E}\left(\left\|\hat{f}_{m}^{X}-f\right\|^{2}\right) \geq\left\|f-f_{m}\right\|^{2}+c^{\prime} \frac{\sqrt{m}}{n}-\frac{\|f\|^{2}}{n} .
$$

Proposition 3.2 in the Hermite case is proved in Belomestny et al. (2017), Proposition 2.2.

3.2. Specific rates under small bias. For some classes of distributions, the rate of the bias term can be much smaller than polynomial. This implies a much better rate of convergence for the $\mathbb{L}^{2}$-risk of estimators.

- Laguerre case. Consider the class of mixtures of exponential distributions defined, for $v>1$, by

$$
\mathcal{E}(v)=\left\{f: f(x)=\int_{0}^{\infty} \theta \exp (-\theta x) d \Pi(\theta), \Pi[1 / v, v]=1\right\}
$$

Proposition 3.3. Let $f \in \mathcal{E}(v)$ for some real number $v>1$ and set $\rho=(v-1 / v+1)^{2}<1$. Then, for $m_{\text {opt }}=[\log n /|\log \rho|]$,

$$
\mathbb{E}\left(\left\|\hat{f}_{m_{o p t}}^{X}-f\right\|\right) \lesssim \sqrt{\log n} / n
$$

- Hermite case. In Belomestny et al. (2017), Section 4.4, we investigated the risk rate of Hermite estimators on classes of mean mixtures or variance mixtures of Gaussian distributions and obtained the following results. Define, for $\phi$ the standard Gaussian density,

$$
\begin{gathered}
\mathcal{F}(C)=\left\{f: f(x)=\phi \star \Pi(x)=\int \phi(x-u) d \Pi(u), \Pi \in \mathcal{P}(C)\right\}, \\
\mathcal{P}(C):=\left\{\Pi \in \mathcal{P}(\mathbb{R}), \Pi(|u|>t) \leq C \exp \left(-t^{2} / C\right) \text { for all positive } t\right\} .
\end{gathered}
$$

For $f \in \mathcal{F}(C)$ and $m_{\mathrm{opt}}=[\log (n)(e C+1 / \log (2))]$, we have $\mathbb{E}\left(\left\|\hat{f}_{m_{\mathrm{opt}}}^{X}-f\right\|^{2}\right) \lesssim \sqrt{\log (n)} / n$. 
Now consider the class of variance mixtures

$$
\mathcal{G}(v)=\left\{f: f(x)=\int_{0}^{+\infty} \frac{\phi(x / u)}{u} d \Pi(u), \Pi([1 / \sqrt{v}, \sqrt{v}])=1\right\}, \quad v>1 .
$$

For $f \in \mathcal{G}(v)$ and $m_{\mathrm{opt}}=[\log (n) /|\log (\rho)|]$, with $\rho$ as above, $\mathbb{E}\left(\left\|\hat{f}_{m_{\mathrm{opt}}}^{X}-f\right\|^{2}\right) \lesssim \sqrt{\log (n)} / n$.

3.3. Adaptive estimation. The density $f$ being unknown, we do not know what kind of regularity it has, therefore, a data-driven choice of the dimension of the projection space has to be done. The interest of the data-driven procedure is that it allows to realize automatically the finite sample bias-variance compromise and also to automatically reach the best possible asymptotic rate without requiring any knowledge on the bias order. The data-driven choice of $m$ mimicks the minimization of the squared bias-variance bound using estimators of the risk bound terms. As $\left\|f-f_{m}\right\|^{2}=\|f\|^{2}-\left\|f_{m}\right\|^{2}$, the squared bias is estimated by $-\left\|\hat{f}_{m}^{X}\right\|^{2}$, getting rid of $\|f\|^{2}$ which is unknown but constant. Thus we set, for $\kappa$ a numerical constant,

$$
\hat{m}_{X}=\arg \min _{m \in\left\{1, \ldots, m_{n}\right\}}\left(-\left\|\hat{f}_{m}^{X}\right\|^{2}+\widehat{\operatorname{pen}}_{X}(m)\right), \widehat{\operatorname{pen}}_{X}(m)=\kappa \frac{\widehat{V}_{m}^{X}}{n}, \widehat{V}_{m}^{X}=\frac{1}{n} \sum_{i=1}^{n} \sum_{j=0}^{m-1} \psi_{j}^{2}\left(X_{i}\right) .
$$

Clearly, $\widehat{V}_{m}^{X}$ is an estimate of $\mathbb{E}\left(\sum_{j=0}^{m-1} \psi_{j}^{2}\left(X_{1}\right)\right)$. We set $\operatorname{pen}_{X}(m):=\mathbb{E}\left(\widehat{\operatorname{pen}}_{X}(m)\right)=\kappa V_{m}^{X} / n$ and prove:

Theorem 3.1. Assume that $m_{n} \leq(n / \log (n))^{\alpha}$ with $\alpha=1$ for Laguerre and $\alpha=6 / 5$ for Hermite, then there exists a numerical value $\kappa_{0}$ such that for all $\kappa \geq \kappa_{0}$,

$$
\mathbb{E}\left(\left\|\hat{f}_{\hat{m}_{X}}^{X}-f\right\|^{2}\right) \leq 3 \inf _{m \in\{1, \ldots, n\}}\left(\left\|f-f_{m}\right\|^{2}+\operatorname{pen}_{X}(m)\right)+\frac{C}{n},
$$

where $C$ is a constant depending on $\|f\|$.

The Hermite case is proved in Belomestny et al. (2017, Theorem 2.1). We prove here the result in the Laguerre case.

Remark 3.1. Note that the risk bound achieves automatically the bias-variance compromise, up to a negligible term of order $O(1 / n)$, and in this sense, $\hat{f}_{\hat{m}_{X}}^{X}$ is adaptive.

\section{Projection estimator of $f$ when $Y_{i}$ 's are observed}

Now, our aim is to build an estimator of $f$ from the observations $Y_{1}, \ldots, Y_{n}$ given by (1).

4.1. Preliminary properties and risk bounds. The estimation of $f$ in model (1) is an inverse problem which can be solved by Hermite or Laguerre projection estimator, depending on the support of the density $f$. The hidden variables $X_{i}$ are either real-valued or nonnegative, with unknown density $f$. The construction of estimators rely on relations between the density $f_{Y}$ and survival function $\bar{F}_{Y}(y)=\mathbb{P}(Y>y)$ of $Y_{i}$ and those of $X_{i}$. We recall these relations:

$$
\begin{gathered}
\forall y \in \mathbb{R}, \quad f_{Y}(y)=\int_{y}^{+\infty} \frac{f(x)}{x} d x \mathbf{I}(y \geq 0)+\int_{-\infty}^{y} \frac{f(x)}{|x|} d x \mathbf{I}(y<0), \\
\forall y \in \mathbb{R}, \quad \bar{F}_{Y}(y)+y f_{Y}(y)=\bar{F}(y) .
\end{gathered}
$$

When $f$ is $\mathbb{R}^{+}$-supported, (13) reduces to $f_{Y}(y)=\int_{y}^{+\infty}(f(u) / u) d u \mathbf{I}_{y \geq 0}$. We can prove: 
Lemma 4.1. (1) Let $t: \mathbb{R} \rightarrow \mathbb{R}$ be bounded, derivable, then

$$
\mathbb{E}\left(t\left(Y_{1}\right)+Y_{1} t^{\prime}\left(Y_{1}\right)\right)=\mathbb{E} t\left(X_{1}\right) .
$$

In particular, for all $j \geq 0$,

$$
a_{j}(f)=\left\langle f, \psi_{j}\right\rangle=\left\langle f_{Y},\left(y \psi_{j}\right)^{\prime}\right\rangle
$$

(2) Assume that $\mathbb{E}\left|X_{1}\right|<+\infty$. Let $t \in \mathbb{L}^{2}(\mathbb{R})$ or $\mathbb{L}^{2}\left(\mathbb{R}^{+}\right)$, then $\mathbb{E}\left(Y_{1}^{2} t^{2}\left(Y_{1}\right)\right) \leq \mathbb{E}\left|X_{1}\right|\|t\|^{2}$.

Equality (15) is the basement of the estimation procedure and leads to (16), which links the coefficients of $f$ and $f_{Y}$ on the Laguerre or Hermite basis. So, using (16), we get for all $j \geq 0$ :

$$
a_{j}(f)=\mathbb{E}\left(\psi_{j}\left(Y_{1}\right)+Y_{1} \psi_{j}^{\prime}\left(Y_{1}\right)\right) .
$$

Therefore, we define a collection of projection estimator of $f$ based on the observation $\left(Y_{1}, \ldots, Y_{n}\right)$ by:

$$
\hat{f}_{m}(x)=\sum_{j=1}^{m} \hat{a}_{j} \psi_{j}(x), \quad \text { with } \hat{a}_{j}=\frac{1}{n} \sum_{i=1}^{n}\left[Y_{i} \psi_{j}^{\prime}\left(Y_{i}\right)+\psi_{j}\left(Y_{i}\right)\right] .
$$

Then we can prove the following risk bound.

Proposition 4.1. Let $\hat{f}_{m}$ be the estimator defined by (18), then we have

$$
\mathbb{E}\left(\left\|\hat{f}_{m}-f\right\|^{2}\right)=\left\|f-f_{m}\right\|^{2}+\frac{V_{m}}{n}-\frac{\left\|f_{m}\right\|^{2}}{n}, \quad V_{m}=\sum_{j=0}^{m-1} \mathbb{E}\left[Y_{1} \psi_{j}^{\prime}\left(Y_{1}\right)+\psi_{j}\left(Y_{1}\right)\right]^{2}
$$

where $V_{m}$ is such that, for $m$ large enough,

$$
V_{m} \leq \mathrm{cm}^{3 / 2}
$$

(1) in the Laguerre case, if $\mathbb{E}\left(X_{1}\right)<+\infty$ and $\mathbb{E}\left(1 / \sqrt{X_{1}}\right)<+\infty$,

(2) in the Hermite case, if $\mathbb{E}\left(\left|X_{1}\right|^{2+2 / 3}\right)<+\infty$,

where $c$ is a constant which does not depend on $m$, but depends on the above moments of $X_{1}$.

Remark 4.1. In the Laguerre case, with no moment condition, we have, for all $m \geq 1, V_{m} \leq$ $\mathrm{Cm}^{3}$ (see Belomestny et al., 2016).

In the Hermite case, if $\mathbb{E}\left(\left|X_{1}\right|\right)<+\infty$, then for all $m \geq 1, V_{m} \leq C m^{2}$ and if $\mathbb{E}\left(X_{1}^{2}\right)<+\infty$, then $V_{m} \leq C m^{11 / 6}$, see the proof in Section 7.10.

We can deduce rates of convergence on Sobolev-Laguerre and Sobolev-Hermite balls.

Proposition 4.2. Assume that $f$ belongs to $W_{L}^{s}(D)$ or to $W_{H}^{s}(D)$ (see (8) and (9)).

If $\mathbb{E}\left(X_{1}\right)<+\infty$ and $\mathbb{E}\left(1 / \sqrt{X_{1}}\right)<+\infty$ in the Laguerre case, or if $\mathbb{E}\left|X_{1}\right|^{2 / 3}<+\infty$ in the Hermite case, then for $m_{\mathrm{opt}}=\left[n^{1 /(s+3 / 2)}\right]$,

$$
\mathbb{E}\left(\left\|\hat{f}_{m_{\mathrm{opt}}}-f\right\|^{2}\right) \lesssim n^{-\frac{2 s}{2 s+3}} .
$$

The results of Section 3.2 apply here. For exponential mixtures in the Laguerre case, or mean or variance Gaussian mixtures in the Hermite case, the bias is exponentially decreasing. Thus, the same choices $m_{\text {opt }}$ yield a rate of order $[\log (n)]^{3 / 2} / n$.

Similarly as above, the bound $O\left(\mathrm{~m}^{3 / 2}\right)$ on the variance term cannot be improved:

Proposition 4.3. (1) Laguerre case: if $\mathbb{E}\left(1 / \sqrt{X_{1}}\right)<+\infty$ and there exist $0<a<b$, with $\inf _{a \leq x \leq b} f(x) \geq c>0$, then, for $m$ large enough, there exists a constant $c_{1}$ such that $V_{m} \geq c_{1} m^{3 / 2}$, where $c_{1}$ does not depend on $m$. 
(2) Hermite case: If there exist $a<b$, with $\inf _{a \leq x \leq b} f(x) \geq c>0$, then, for $m$ large enough, there exists a constant $c_{2}$ such that $V_{m} \geq c_{2} m^{3 / 2}$, where $c_{2}$ does not depend on $m$.

Consequently in each case, for $c^{\star}=c_{1}$ or $c_{2}$, we have

$$
\mathbb{E}\left(\left\|\hat{f}_{m}-f\right\|^{2}\right) \geq\left\|f-f_{m}\right\|^{2}+c^{\star} \frac{m^{3 / 2}}{n}-\frac{\|f\|^{2}}{n} .
$$

4.2. Matrix representations. Now, we give formulae providing the links between the coefficients of $f$ and those of $f_{Y}$ on basis $\left(\psi_{j}\right)_{0 \leq j \leq m-1}$ and allowing to build easily the estimators. For $g$ a function, we denote $\vec{a}_{k}(g):={ }^{t}\left(a_{0}(g), \ldots, a_{k-1}(g)\right), k \geq 1$, where $a_{j}(g)=\left\langle g, \psi_{j}\right\rangle$.

- Specific Laguerre formula. Using formula (47) (see Section 7.1), we get $a_{0}(f)=(1 / 2) a_{0}\left(f_{Y}\right)+$ $(1 / 2) a_{1}\left(f_{Y}\right)$ and for $j \geq 1$,

$$
a_{j}(f)=-\frac{j}{2} a_{j-1}\left(f_{Y}\right)+\frac{1}{2} a_{j}\left(f_{Y}\right)+\frac{j+1}{2} a_{j+1}\left(f_{Y}\right) .
$$

Introducing the matrix $\mathbf{H}_{m}=\left(\left[\mathbf{H}_{m}\right]_{k, \ell}\right)_{1 \leq k, \ell \leq m}$ with size $m \times(m+1)$ given by $\left[\mathbf{H}_{m}\right]_{k, \ell}=0$ if $\ell \neq k-1, k, k+1$ and $\left[\mathbf{H}_{m}\right]_{1,1}=1 / 2,\left[\mathbf{H}_{m}\right]_{1,2}=1 / 2$ and for $k \geq 2$,

$$
\left[\mathbf{H}_{m}\right]_{k, k-1}=-\frac{k-1}{2}, \quad\left[\mathbf{H}_{m}\right]_{k, k}=\frac{1}{2}, \quad\left[\mathbf{H}_{m}\right]_{k, k+1}=\frac{k}{2},
$$

yields the linear relation between the vectors of coefficients of $f$ and $f_{Y}: \vec{a}_{m-1}(f)=\mathbf{H}_{m} \vec{a}_{m}\left(f_{Y}\right)$. We thus have for $m \geq 1$, setting $\overrightarrow{\hat{a}}_{m-1}={ }^{t}\left(\hat{a}_{j}\right)_{0 \leq j \leq m-1}, \quad \overrightarrow{\hat{a}}_{m}(Y)={ }^{t}\left(\hat{a}_{j}(Y)\right)_{0 \leq j \leq m}$, the following relation which is convenient to compute the estimator

$$
\overrightarrow{\hat{a}}_{m-1}=\mathbf{H}_{m} \overrightarrow{\hat{a}}_{m}(Y) \quad \text { with } \quad \hat{a}_{j}(Y):=\frac{1}{n} \sum_{i=1}^{n} \varphi_{j}\left(Y_{i}\right) .
$$

- Specific Hermite formula. From $x f_{Y}^{\prime}(x)=-f(x)$ and the relations (52), we get

$$
\left\{\begin{array}{l}
a_{0}(f)=(1 / 2)\left(a_{0}\left(f_{Y}\right)-\sqrt{2} a_{2}\left(f_{Y}\right)\right), \quad a_{1}(f)=(1 / 2)\left(a_{1}\left(f_{Y}\right)-\sqrt{6} a_{3}\left(f_{Y}\right)\right) \\
a_{j}(f)=(1 / 2)\left(a_{j}\left(f_{Y}\right)-\sqrt{(j+1)(j+2)} a_{j+2}\left(f_{Y}\right)+\sqrt{(j-1) j} a_{j-2}\left(f_{Y}\right)\right), \quad j \geq 2 .
\end{array}\right.
$$

Therefore $\vec{a}_{m}(f)=\mathbf{A}_{m, m+2} \vec{a}_{m+2}\left(f_{Y}\right)$, where $\mathbf{A}_{m, m+2}$ is the $m \times(m+2)$ matrix deduced from the above relations. So, for $m \geq 1$, setting $\overrightarrow{\hat{a}}_{m}={ }^{t}\left(\hat{a}_{j}\right)_{0 \leq j \leq m-1}, \overrightarrow{\hat{a}}_{m+2}(Y)={ }^{t}\left(\hat{a}_{j}(Y)\right)_{0 \leq j \leq m+1}$, gives the following relation:

$$
\overrightarrow{\hat{a}}_{m}=\mathbf{A}_{m, m+2} \overrightarrow{\hat{a}}_{m+2}(Y) \quad \text { with } \quad \hat{a}_{j}(Y):=\frac{1}{n} \sum_{i=1}^{n} h_{j}\left(Y_{i}\right) .
$$

4.3. Model selection. Define $\mathcal{M}_{n}=\left\{1, \ldots, m_{n}\right\}$, where $m_{n}$ is the largest integer of the collection and set

$$
\begin{gathered}
\hat{m}=\arg \min _{m \in \mathcal{M}_{n}}\left\{-\left\|\hat{f}_{m}\right\|^{2}+\widehat{\operatorname{pen}}(m)\right\}, \quad \widehat{\operatorname{pen}}(m)=\kappa \frac{\widehat{V}_{m}}{n}, \\
\widehat{V}_{m}=\frac{1}{n} \sum_{i=1}^{n} \sum_{j=0}^{m-1}\left[Y_{i} \psi_{j}^{\prime}\left(Y_{i}\right)+\psi_{j}\left(X_{i}\right)\right]^{2},
\end{gathered}
$$

where $\kappa$ is a numerical constant. Note that $\widehat{V}_{m}$ is an estimate of $V_{m}$ such that $\mathbb{E}\left(\widehat{V}_{m}\right)=V_{m}$. We denote $\operatorname{pen}(m)=\mathbb{E}(\widehat{\operatorname{pen}}(m))=\kappa V_{m} / n$. 
Theorem 4.1. Assume that, in the Hermite case, $\mathbb{E}\left(\left|X_{1}\right|\right)<+\infty$ and $\inf _{a \leq x \leq b} f(x)>0$ for some interval $[a, b]$ and in the Laguerre case, $\mathbb{E}\left(1 / \sqrt{X_{1}}\right)<+\infty$ and $\inf _{a \leq x \leq b} f(x)>0$ for some interval $[a, b]$ with $0<a<b$. Assume also that the collection of models is such that

$$
m_{n} \leq n^{\beta} \text { with } \beta=1 / 3 \text { for Laguerre case, } \beta=6 / 17 \text { for Hermite case. }
$$

Then there exists a numerical constant $\kappa_{0}$ such that, for $\kappa \geq \kappa_{0}$, the estimator $\hat{f}_{\hat{m}}$ where $\hat{m}$ is defined by (23) satisfies

$$
\mathbb{E}\left(\left\|\hat{f}_{\hat{m}}-f\right\|^{2}\right) \leq C \inf _{m \in \mathcal{M}_{n}}\left(\left\|f-f_{m}\right\|^{2}+\kappa \frac{V_{m}}{n}\right)+C^{\prime} \frac{\log (n)}{n},
$$

where $C$ is a numerical constant $(C=4$ suits $)$ and $C^{\prime}$ is a constant depending on $\|f\|_{\infty}$.

Note that Remark 3.1 applies here also. The restriction (24) implies that the optimal order of $m$, for $f$ in a Sobolev ball, can be reached only if the function is regular enough i.e. $s$ large enough. More precisely, under the assumptions of Theorem 4.1 and of Proposition 4.2, we have, for $f \in W_{L}^{s}(D)$ (Laguerre case) that $m_{\mathrm{opt}}=\left[n^{1 /(s+(3 / 2))}\right]$ can be reached in the model collection if $s>3 / 2\left(n^{1 /(s+3 / 2)}<n^{1 / 3}\right)$. For $f \in W_{H}^{s}(D)$ (Hermite case), $m_{\text {opt }}$ is reached for $s>4 / 3$ $\left(n^{1 /(s+3 / 2)}<n^{6 / 17}\right)$.

4.4. Deconvolution estimator based on multiplicative censored observations. In Belomestny et al. (2017), for the case of direct observations $X_{1}, \ldots, X_{n}$, projection estimators on the Hermite basis are compared to projection estimators on the sine cardinal basis. The comparison is relevant as Sobolev Hermite spaces with regularity index $s$ are included in usual Sobolev spaces with the same regularity index (see Appendix A). For direct observations, the two estimators are proved in Belomestny et al. (2017) to be asymptotically equivalent. We prove here that it is also the case in the multiplicative censored case.

Let us recall the definition of the sine cardinal basis. For $u, v \in \mathbb{L}^{1}(\mathbb{R}) \cap \mathbb{L}^{2}(\mathbb{R})$, we denote by $u^{*}(t)=\int e^{i t x} u(x) d x$ and $\langle u, v\rangle=\int u \bar{v}=(2 \pi)^{-1}\left\langle u^{*}, v^{*}\right\rangle$. We recall that $u^{* *}(\cdot)=2 \pi u(-\cdot)$. Let $\theta(x)=\sin (\pi x) /(\pi x)$ which satisfies $\theta^{*}(t)=\mathbf{I}_{[-\pi, \pi]}(t)$, where $\theta^{*}$ denotes the Fourier transform of $\theta$. The functions $\left(\theta_{\ell, j}(x)=\sqrt{\ell} \theta(\ell x-j), j \in \mathbb{Z}\right)$ constitute an orthonormal system in $\mathbb{L}^{2}(\mathbb{R})$. The space $F_{\ell}$ generated by this system is exactly the subspace of $\mathbb{L}^{2}(\mathbb{R})$ of functions having Fourier transforms with compact support $[-\pi \ell, \pi \ell]$. The orthogonal projection $\bar{f}_{\ell}$ of $f$ on $F_{\ell}$ satisfies $\bar{f}_{\ell}^{*}=f^{*} \mathbf{I}_{[-\pi \ell, \pi \ell]}$ and

$$
\bar{f}_{\ell}(x)=\frac{1}{2 \pi} \int_{-\pi \ell}^{\pi \ell} e^{-i t x} f^{*}(t) d t=\sum_{\ell \in \mathbb{Z}} a_{\ell, j} \theta_{\ell, j}, \quad a_{\ell, j}=\left\langle f, \theta_{\ell, j}\right\rangle .
$$

Note that

$$
\left\|f-\bar{f}_{\ell}\right\|^{2}=\frac{1}{2 \pi} \int_{|t| \geq \pi \ell}\left|f^{*}(t)\right|^{2} d t .
$$

Now, relation (15) holds for $t(x)=e^{i u x}: \mathbb{E}\left(e^{i u Y_{1}}+i u Y_{1} e^{i u Y_{1}}\right)=\mathbb{E} e^{i u X_{1}}$. Therefore, we can estimate the Fourier transform $f^{*}(u)=\int e^{i u x} f(x) d x$ of $f$ by

$$
\frac{1}{n} \sum_{k=1}^{n}\left(e^{i u Y_{k}}+i u Y_{k} e^{i u Y_{k}}\right)
$$

This yields the following new estimator of $f$, of deconvolution type:

$$
\widetilde{f}_{\ell}(x)=\frac{1}{2 \pi} \int_{-\pi \ell}^{\pi \ell} e^{-i u x} \frac{1}{n} \sum_{k=1}^{n}\left(e^{i u Y_{k}}+i u Y_{k} e^{i u Y_{k}}\right) d u,
$$


which is an unbiased estimator of $\bar{f}_{\ell}$ given in (25). We can integrate and obtain:

$$
\tilde{f}_{\ell}(x)=\frac{1}{n} \sum_{k=1}^{n}(-x) \frac{\sin \left(\pi \ell\left(Y_{k}-x\right)\right)}{\pi\left(Y_{k}-x\right)^{2}}+\frac{Y_{k}}{Y_{k}-x} \ell \cos \left(\pi \ell\left(Y_{k}-x\right)\right) .
$$

Alternatively, using a truncated series expansion gives an approximation of the estimator which may be easier to compute in practice. Let

$$
\widetilde{f}_{\ell}^{(n)}(x)=\sum_{|j| \leq L_{n}} \tilde{a}_{\ell, j} \theta_{\ell, j}(x) \quad \text { where } \quad \tilde{a}_{\ell, j}=\frac{1}{n} \sum_{k=1}^{n} \theta_{\ell, j}\left(Y_{k}\right)+Y_{k} \theta_{\ell, j}^{\prime}\left(Y_{k}\right) .
$$

We can prove:

Proposition 4.4. Assume that $\mathbb{E}\left(X_{1}^{2}\right)<+\infty$. The estimator $\tilde{f}_{\ell}$ satisfies

$$
\mathbb{E}\left(\left\|\widetilde{f}_{\ell}-f\right\|^{2}\right) \leq\left\|f-\bar{f}_{\ell}\right\|^{2}+\frac{\pi^{2} \ell^{3} \mathbb{E} Y_{1}^{2}}{3 n}+\frac{\ell}{n} .
$$

If moreover $M_{2}=\int x^{2} f^{2}(x) d x<+\infty$,

$$
\mathbb{E}\left(\left\|\widetilde{f}_{\ell}^{(n)}-f\right\|^{2}\right) \leq\left\|f-\bar{f}_{\ell}\right\|^{2}+\frac{\ell}{n}\left(1+\mathbb{E} Y_{1}^{2} \frac{\pi^{2} \ell^{2}}{3}\right)+4\left(M_{2}+1\right) \frac{\ell^{2}}{L_{n}} .
$$

As we consider estimators $\widetilde{f}_{\ell}$ with bounded variance, we will impose $\ell^{3} \leq n$. Consequently, if $L_{n} \geq n$, the last residual term $\ell^{2} / L_{n}$ is of order $O\left(\ell^{2} / n\right)$ and thus less than the variance term $\ell^{3} / n$; if $L_{n} \geq n^{5 / 3}$, it is of order $O(1 / n)$ and thus negligible.

In this context, Sobolev balls are defined by

$$
\mathcal{W}^{s}(L)=\left\{f \in \mathbb{L}^{2}(\mathbb{R}), \quad \int_{\mathbb{R}}\left(1+t^{2 s}\right)\left|f^{*}(t)\right|^{2} d t \leq L<+\infty\right\} .
$$

It is easy to see that, if $f \in \mathcal{W}^{s}(L)$, then, from (26), we have $\left\|f-\bar{f}_{\ell}\right\| \leq L \ell^{-2 s}$. Choosing $\ell_{\text {opt }}=c n^{-1 /(2 s+3)}$, implies, from (30), that $\mathbb{E}\left(\left\|\widetilde{f}_{\ell_{\mathrm{opt}}}-f\right\|^{2}\right) \leq C n^{-2 s /(2 s+3)}$. This optimal rate is identical to the rate obtained for $\hat{f}_{m_{\mathrm{opt}}}$ under $\mathbb{E}\left(\left|X_{1}\right|^{2+2 / 3}\right)<+\infty$. Consequently, the deconvolution and Hermite estimators are asymptotically equivalent (with $\ell=\sqrt{m}$ ). However, from the computational efficiency point of view, the Hermite estimator is to be preferred. In Belomestny et al. (2017), Section 4.5, a notion of complexity is defined. Hermite estimators are proved to have much lower complexity than deconvolution estimators in the case of direct observations. Analogous computations of complexity in the present indirect observations case lead to the same conclusions.

\section{Projection estimator of $f$ When $Z_{i}=X_{i}+\Sigma_{i}$ ARE ObServed}

5.1. Estimation strategy in the Laguerre case. We consider Model (2) where $X_{i}$ and $\Sigma_{i}$ are nonnegative. It holds that

$$
f_{Z}(x)=f \star f_{\Sigma}(x)=\int_{0}^{x} f(u) f_{\Sigma}(x-u) d u .
$$

The convolution property of the Laguerre functions $\left(\varphi_{j}\right)_{j}$ given in formula (49) allows to write

$$
\begin{aligned}
\sum_{k=0}^{\infty} a_{k}\left(f_{Z}\right) \varphi_{k}(x) & =\sum_{j=0}^{+\infty} \sum_{k=0}^{+\infty} a_{j}(f) a_{k}\left(f_{\Sigma}\right) \varphi_{j} \star \varphi_{k}(x) \\
& =\sum_{k=0}^{\infty} \varphi_{k}(x) \sum_{\ell=0}^{k} 2^{-1 / 2}\left(a_{k-\ell}\left(f_{\Sigma}\right)-a_{k-\ell-1}\left(f_{\Sigma}\right)\right) a_{\ell}(f),
\end{aligned}
$$


with convention $a_{k}(f)=0$ if $k<0$.

Define the $m \times m$ triangular matrix $\boldsymbol{\Sigma}_{m}=\left(\sigma_{i, j}\right)_{0 \leq i, j \leq m-1}$ where

$$
\sigma_{i, j}=2^{-1 / 2}\left(\left\langle f_{\Sigma}, \varphi_{i-j}\right\rangle \mathbf{I}_{i-j \geq 0}-\left\langle f_{\Sigma}, \varphi_{i-j-1}\right\rangle \mathbf{I}_{i-j-1 \geq 0}\right) .
$$

As $\sigma_{i, j}=\sigma(i-j) \mathbf{I}_{i-j \geq 0}, \boldsymbol{\Sigma}_{m}$ is a Toeplitz triangular matrix with diagonal elements $\sigma_{i, i}=$ $2^{-1 / 2}\left\langle f_{\Sigma}, \varphi_{0}\right\rangle>0$. It is thus invertible and for all $m \geq 1$,

$$
\vec{a}_{m}\left(f_{Y}\right)={ }^{t}\left(a_{j}(f)\right)_{0 \leq j \leq m-1}=\boldsymbol{\Sigma}_{m}^{-1}\left[\left(a_{j}\left(f_{Z}\right)\right)_{0 \leq j \leq m-1}\right]=\boldsymbol{\Sigma}_{m}^{-1} \vec{a}_{m}\left(f_{Z}\right),
$$

The projection estimator of $f$ on $S_{m}$ based on $\left(Z_{1}, \ldots, Z_{n}\right)$ is given by

$$
\tilde{f}_{m}=\sum_{j=0}^{m-1} \tilde{a}_{j} \varphi_{j}, \quad \overrightarrow{\tilde{a}}_{m}={ }^{t}\left(\tilde{a}_{j}\right)_{0 \leq j \leq m-1}=\boldsymbol{\Sigma}_{m}^{-1} \overrightarrow{\hat{a}}_{m}(Z), \quad m \geq 1
$$

where $\overrightarrow{\hat{a}}_{m}(Z)=\left[\left(\hat{a}_{j}(Z)\right)_{0 \leq j \leq m-1}\right]$ and $\hat{a}_{j}(Z)$ is defined by

$$
\hat{a}_{j}(Z):=\frac{1}{n} \sum_{i=1}^{n} \varphi_{j}\left(Z_{i}\right) .
$$

This estimator is proposed in Mabon (2017) and inspired from Comte et al. (2017). The following risk bound is an improvement of the bound given in Mabon (2017).

Proposition 5.1. Assume that $\left\|f_{\Sigma}\right\|_{\infty}<+\infty$ and either $\mathbb{E}\left(X_{1}^{-1 / 2}\right)<+\infty$ or $\mathbb{E}\left(\Sigma_{1}^{-1 / 2}\right)<+\infty$. Let $\tilde{f}_{m}$ be given by (35). Then we have

$$
\mathbb{E}\left(\left\|\tilde{f}_{m}-f\right\|^{2}\right) \leq\left\|f-f_{m}\right\|^{2}+\frac{\left[c \sqrt{m}\left\|\boldsymbol{\Sigma}_{m}^{-1}\right\|_{\text {op }}^{2}\right] \wedge\left[\left\|f_{\Sigma}\right\|_{\infty}\left\|\boldsymbol{\Sigma}_{m}^{-1}\right\|_{F}^{2}\right]}{n}
$$

where $\|\mathbf{A}\|_{F}^{2}=\operatorname{Tr}\left({ }^{t} \mathbf{A} \mathbf{A}\right)$ ( $\operatorname{Tr}$ denotes the trace of the matrix) and $\|\mathbf{A}\|_{\text {op }}^{2}=\lambda_{\max }\left({ }^{t} \mathbf{A} \mathbf{A}\right)$ is the maximal eigenvalue of ${ }^{t} \mathbf{A} \mathbf{A}$.

The bias term is unchanged. The variance term is increasing in $m$ because of the special form of $\boldsymbol{\Sigma}_{m}^{-1}$ (lower triangular and Toeplitz, see Mabon (2017)). If $\Sigma_{i}=0$ and thus $\boldsymbol{\Sigma}_{m}=I d$, then $\left\|\boldsymbol{\Sigma}_{m}^{-1}\right\|_{\text {op }}^{2}=1$ and $\left\|\boldsymbol{\Sigma}_{m}^{-1}\right\|_{F}^{2}=m$ thus the variance term is of order $\sqrt{m} / n$ as expected.

In Comte et al. (2017), the order of $\left\|\boldsymbol{\Sigma}_{m}^{-1}\right\|_{F}^{2}$ in function of $m$ is studied. In particular, if $\Sigma_{i}$ has a Gamma distribution $\Gamma(r, \lambda), r \in \mathbb{N}, r \geq 1$, there exist constants $c, C$ such that

$$
c m^{2 r} \leq\left\|\boldsymbol{\Sigma}_{m}^{-1}\right\|_{\mathrm{op}}^{2} \leq\left\|\boldsymbol{\Sigma}_{m}^{-1}\right\|_{F}^{2} \leq C m^{2 r} .
$$

Therefore the following corollary holds:

Corollary 5.1. Assume that $f \in W_{L}^{s}(D)$, and that $\Sigma_{i}$ has a Gamma distribution $\Gamma(r, \lambda), r$ integer, $r \geq 1$. Then $\tilde{f}_{m}$ given by (35) satisfies, for $m_{\text {opt }}=\left[n^{2 r+s}\right]$

$$
\mathbb{E}\left(\left\|\tilde{f}_{m_{\text {opt }}}-f\right\|^{2}\right) \leq C(s, D) n^{-s /(2 r+s)} .
$$

The matrix $\boldsymbol{\Sigma}_{m}$ in the case of gamma noise with integer tail parameter can be computed explicitely: we give it for $r=1,2$ hereafter.

Remark 5.1. $\quad$ For $\Sigma_{1} \sim \mathcal{E}(\lambda)=\gamma(1, \lambda)$, we have $\left[\boldsymbol{\Sigma}_{m}\right]_{i, i}=\lambda /(1+\lambda)$ and

$$
\left[\boldsymbol{\Sigma}_{m}\right]_{i, j}=-2 \lambda \frac{(\lambda-1)^{i-j-1}}{(\lambda+1)^{(i-j+1)}} \text { if } j<i \quad \text { and }\left[\boldsymbol{\Sigma}_{m}\right]_{i, j}=0 \text { otherwise. }
$$

We can compute $\left[\boldsymbol{\Sigma}_{m}^{-1}\right]_{i, j}=(\lambda+1) / \lambda$ if $i=j, 2 / \lambda$ if $i>j$ and 0 otherwise. Note that

$$
\left\|\boldsymbol{\Sigma}_{m}^{-1}\right\|_{F}^{2}=2 \frac{m^{2}}{\lambda^{2}}+m\left(1+\frac{2}{\lambda}-\frac{1}{\lambda^{2}}\right) .
$$


- For $\Sigma_{1} \sim \Gamma(2, \mu)$, we have $\left[\boldsymbol{\Sigma}_{m}\right]_{i, i}=(\mu /(1+\mu))^{2},\left[\boldsymbol{\Sigma}_{m}\right]_{i+1, i}=-4 \mu^{2} /(1+\mu)^{3}$ and

$$
\left[\boldsymbol{\Sigma}_{m}\right]_{i, j}=4(i-j-\mu) \mu^{2} \frac{(\mu-1)^{i-j-2}}{(\mu+1)^{i-j+2}} \text { if } i>j+1 \quad \text { and }\left[\boldsymbol{\Sigma}_{m}\right]_{i, j}=0 \text { otherwise. }
$$

We can propose a method to select $m$ automatically, aiming at a data driven bias variance compromise and simplifying Mabon (2017). We define

$$
\tilde{m}=\arg \min _{m \in \mathcal{M}_{n}}\left(-\left\|\tilde{f}_{m}\right\|^{2}+\widetilde{\operatorname{pen}}(m)\right) \quad \text { with } \widetilde{\operatorname{pen}}(m)=\kappa \frac{\log \left(2+\left\|\boldsymbol{\Sigma}_{m}^{-1}\right\|_{F}^{2}\right)\left\|\boldsymbol{\Sigma}_{m}^{-1}\right\|_{F}^{2}}{n}
$$

where

$$
\mathcal{M}_{n}=\left\{m \in \mathbb{N}^{*}, m \leq n / \log (2+n),\left\|\boldsymbol{\Sigma}_{m}^{-1}\right\|_{F}^{2} \leq n\right\} .
$$

Theorem 5.1. Let $\tilde{f}_{m}$ be given by (35) and $\tilde{m}$ by (39). There exists a numerical constant $\kappa_{0}$ such that for any $\kappa \geq \kappa_{0}$, we have

$$
\mathbb{E}\left(\left\|\tilde{f}_{\tilde{m}}-f\right\|^{2}\right) \leq C_{1} \inf _{m \in \mathcal{M}_{n}}\left(\left\|f-f_{m}\right\|^{2}+\widetilde{\operatorname{pen}}(m)\right)+\frac{C_{2}}{n} .
$$

In the penalty, we have chosen $\left\|\boldsymbol{\Sigma}_{m}^{-1}\right\|_{F}^{2}$ rather than $\sqrt{m}\left\|\boldsymbol{\Sigma}_{m}^{-1}\right\|_{\text {op }}^{2}$, which is easier to handle.

5.2. Estimation strategy in the Hermite case. We consider Model (2) where now $\left(X_{i}\right)_{1 \leq i \leq n}$ and $\left(\Sigma_{i}\right)_{1 \leq i \leq n}$ are real valued. This model is classically dealt with by Fourier deconvolution, and yields the following estimator

$$
f_{\ell}^{\diamond}(x):=\frac{1}{2 \pi} \int_{-\pi \ell}^{\pi \ell} e^{-i t x} \frac{\widehat{f_{Z}^{*}}(t)}{f_{\Sigma}^{*}(-t)} d t \quad \text { where } \quad \widehat{f_{Z}^{*}}(t)=\frac{1}{n} \sum_{k=1}^{n} e^{i t Z_{k}}
$$

is the empirical characteristic function of $Z$.

Here we propose projection estimators on the Hermite basis. We use that $h_{j}^{*}(t)=\sqrt{2 \pi} i^{j} h_{j}(t)$ and write that $f_{Z}=f \star f_{\Sigma}$ and thus $f_{Z}^{*}=f^{*} f_{\Sigma}^{*}$, where $f^{*}=\sqrt{2 \pi} \sum_{j \geq 0} a_{j}(f) i^{j} h_{j}$. Therefore,

$$
a_{j}(f)=\frac{(-i)^{k}}{\sqrt{2 \pi}}\left\langle f_{Z}^{*}, \frac{h_{j}}{f_{\Sigma}^{*}(-.)}\right\rangle .
$$

This leads to define the estimator of $a_{j}(f)$ as follows:

$$
\check{a}_{j, \ell}=\frac{(-i)^{j}}{\sqrt{2 \pi}} \int_{-\pi \ell}^{\pi \ell} \widehat{f_{Z}^{*}}(t) \frac{h_{j}(t)}{f_{\Sigma}^{*}(t)} d t
$$

and the projection estimator of $f$ as

$$
\check{f}_{m}(x)=\sum_{j=0}^{m-1} \check{a}_{j, \sqrt{m}} h_{j}(x) .
$$

Note that we have chosen $\ell=\sqrt{m}$ in (40). The following risk bound holds.

Proposition 5.2. Consider $\check{f}_{m}$ given by (41) and (40). Then

$$
\mathbb{E}\left(\left\|\check{f}_{m}-f\right\|^{2}\right) \leq \sum_{j \geq m} a_{j}^{2}(f)+\frac{1}{2 \pi} \int_{|t| \geq \pi \sqrt{m}}\left|f^{*}(t)\right|^{2} d t+\frac{1}{2 \pi n} \int_{-\pi \sqrt{m}}^{\pi \sqrt{m}} \frac{1}{\left|f_{\Sigma}^{*}(t)\right|^{2}} d t .
$$


Thus, if $f \in W_{H}^{s}(L) \subset \mathcal{W}^{s}\left(L^{\prime}\right)$ (see (9) and (32) and Appendix A), then the two bias terms in Proposition 5.2 have the same order:

$$
\sum_{j \geq m} a_{j}^{2}(f)+\frac{1}{2 \pi} \int_{|t| \geq \pi \sqrt{m}}\left|f^{*}(t)\right|^{2} d t \leq L m^{-s}+\frac{L^{\prime}}{2 \pi}(\pi \sqrt{m})^{-2 s}=C m^{-s} .
$$

For comparison,

$$
\mathbb{E}\left(\left\|f_{\sqrt{m}}^{\diamond}-f\right\|^{2}\right) \leq \frac{1}{2 \pi} \int_{|t| \geq \pi \sqrt{m}}\left|f^{*}(t)\right|^{2} d t+\frac{1}{2 \pi n} \int_{-\pi \sqrt{m}}^{\pi \sqrt{m}} \frac{1}{\left|f_{\Sigma}^{*}(t)\right|^{2}} d t .
$$

This shows that the two estimators have asymptotically the same rate. The deconvolution estimator has a smaller risk bound. However, in terms of computational efficiency, as described in Belomestny al. (2017), the Hermite estimator is to be preferred as it has lower complexity. For sake of brevity, we do not develop the adaptive procedure.

\section{EXTENSIONS AND CONCLUDING REMARKS}

In this paper, the use of a Laguerre basis to estimate a function $f \in \mathbb{L}^{2}\left(\mathbb{R}^{+}\right)$or a Hermite basis to estimate a function $f \in \mathbb{L}^{2}(\mathbb{R})$ is illustrated in examples of inverse problems. Projection estimators which are easy to implement are built and studied. Data-driven choices of the projection dimension can be proposed leading to adaptive estimators.

Using formulae of Section 7.1.2, the estimation of a function $f \in \mathbb{L}^{2}\left(\mathbb{R}^{+}\right)$by projection estima-

tors on a Laguerre basis $\left(\varphi_{j}^{(\delta)}\right)$ for all $\delta>-1$ is possible for direct observations or multiplicative censored observations. Thanks to Lemma 7.2, we can obtain risk bounds of the same type. In the case of multiplicative censoring, setting $a_{j}^{(\delta)}(f)=\left\langle f, \varphi_{j}^{(\delta)}\right\rangle_{+}$, and using relation (50), we obtain:

$$
a_{j}^{(\delta)}(f)=\frac{\sqrt{(j+1)(j+\delta+1)}}{2} a_{j+1}^{(\delta)}\left(f_{Y}\right)+\frac{1}{2} a_{j}^{(\delta)}\left(f_{Y}\right)-\frac{\sqrt{j(j+\delta)}}{2} a_{j-1}^{(\delta)}\left(f_{Y}\right) .
$$

This allows to define analogously a matrix $\mathbf{H}_{m}^{(\delta)}$ which helps practical computing of the projection estimator. On the contrary, except for $\delta=0$, the bases $\left(\varphi_{j}^{(\delta)}\right)$ do not seem fitted to the deconvolution setting of densities on $\mathbb{R}^{+}$.

An extension of the results presented here could be to study the combination of models (1) and (2) and estimate $f$, the density of $X_{i}$ if observations are either $\left(X_{i}+\Sigma_{i}\right) U_{i}$ or $X_{i} U_{i}+\Sigma_{i}$.

\section{Proofs}

7.1. Formulae for Laguerre functions. The Laguerre polynomial with index $\delta, \delta>-1$, and degree $k$ is given by

$$
L_{k}^{(\delta)}(x)=\frac{1}{k !} e^{x} x^{-\delta} \frac{d^{k}}{d x^{k}}\left(x^{\delta+k} e^{-x}\right)=\sum_{j=0}^{k}\left(\begin{array}{l}
k+\delta \\
k-j
\end{array}\right) \frac{(-x)^{j}}{j !} .
$$

The following holds:

$$
\left(L_{k}^{(\delta)}(x)\right)^{\prime}=-L_{k-1}^{(\delta+1)}(x), \quad \text { for } k \geq 1, \text { and } \int_{0}^{+\infty}\left(L_{k}^{(\delta)}(x)\right)^{2} x^{\delta} e^{-x} d x=\frac{\Gamma(k+\alpha+1)}{k !} .
$$


The sequence $\left(\phi_{k}^{(\delta)}(x)=L_{k}^{(\delta)}(x) x^{\delta / 2} e^{-x / 2}\left(\frac{k !}{\Gamma(k+\alpha+1}\right)^{1 / 2}\right)$ is an orthonormal basis of $\mathbb{L}^{2}\left(\mathbb{R}^{+}\right)$. In this paper, we rather consider the Laguerre functions with index $\delta$, given by

$$
\varphi_{k}^{(\delta)}(x)=\sqrt{2} \phi_{k}^{(\delta)}(2 x)=2^{(\delta+1) / 2}\left(\frac{k !}{\Gamma(k+\delta+1)}\right)^{1 / 2} L_{k}^{(\delta)}(2 x) e^{-x} x^{\delta / 2}
$$

which are more convenient for computing derivatives or integrals of the basis functions especially when $\delta=0$. The family $\left(\varphi_{k}^{(\delta)}\right)_{k \geq 0}$ is an orthonormal basis of $\mathbb{L}^{2}\left(\mathbb{R}^{+}\right)$.

7.1.1. Formulae for $\delta=0$. For $\delta=0$, we set $L_{k}^{(0)}=L_{k}, \varphi_{k}^{(0)}=\varphi_{k}$. Formula (22.7.12) in Abramowitz and Stegun (1964) states that

$$
x L_{j}(x)=-(j+1) L_{j+1}(x)+(2 j+1) L_{j}(x)-j L_{j-1}(x), \quad x L_{j}^{\prime}(x)=j\left(L_{j}(x)-L_{j-1}(x)\right) .
$$

implying

$$
\begin{gathered}
x \varphi_{j}(x)=-\frac{j+1}{2} \varphi_{j+1}(x)+\left(j+\frac{1}{2}\right) \varphi_{j}(x)-\frac{j}{2} \varphi_{j-1}(x), \\
\left(x \varphi_{j}(x)\right)^{\prime}=\varphi_{j}(x)+x \varphi_{j}^{\prime}(x)=-\frac{j}{2} \varphi_{j-1}(x)+\frac{1}{2} \varphi_{j}(x)+\frac{j+1}{2} \varphi_{j+1}(x) .
\end{gathered}
$$

Using (43), we obtain for $j \geq 1$ :

$$
\varphi_{j}^{\prime}(x)=-\varphi_{j}(x)-\sqrt{\frac{2 j}{x}} \varphi_{j-1}^{(1)}(x) .
$$

The following convolution property (formula 22.13.14 in Abramowitz and Stegun (1964)) makes the Laguerre basis relevant in the deconvolution setting

$$
\varphi_{k} \star \varphi_{j}(x)=\int_{0}^{x} \varphi_{k}(u) \varphi_{j}(x-u) d u=2^{-1 / 2}\left(\varphi_{k+j}(x)-\varphi_{k+j+1}(x)\right)
$$

where $\star$ stands for the convolution product.

Lemma 7.1. For all $x \geq 0, \varphi_{0}^{\prime}(x)=-\varphi_{0}(x), \quad \varphi_{j}^{\prime}(x)=-\varphi_{j}(x)-2 \sum_{k=0}^{j-1} \varphi_{k}(x), j \geq 1$. Moreover, $\left|\varphi_{j}^{\prime}(x)\right| \leq \sqrt{2}(2 j+1) \leq 2 \sqrt{2}(j+1), \quad\left|x \varphi_{j}^{\prime}(x)+\varphi_{j}(x)\right| \leq \sqrt{2}(j+1)$.

Proof of Lemma 7.1. The following equality holds $\varphi_{j}^{\prime}(x)=-\varphi_{j}(x)+2 \sqrt{2} e^{-x} L_{j}^{\prime}(2 x)$ which is a polynomial function of degree $j$ multiplied by $e^{-x}$. Thus, it can be decomposed as $\varphi_{j}^{\prime}(x)=$ $\sum_{k=0}^{j} a_{k}^{(j)} \varphi_{k}(x)$ with

$$
\begin{aligned}
a_{k}^{(j)} & =\left\langle\varphi_{j}^{\prime}, \varphi_{k}\right\rangle=\int_{0}^{+\infty} \varphi_{j}^{\prime}(x) \varphi_{k}(x) d x=\left[\varphi_{j}(x) \varphi_{k}(x)\right]_{0}^{+\infty}-\int_{0}^{+\infty} \varphi_{j}(x) \varphi_{k}^{\prime}(x) d x \\
& =-\varphi_{j}(0) \varphi_{k}(0)-\int_{0}^{+\infty} \varphi_{j}(x) \varphi_{k}^{\prime}(x) d x=-2-\left\langle\varphi_{j}, \varphi_{k}^{\prime}\right\rangle=-2-a_{j}^{(k)}
\end{aligned}
$$


Notice that this formula is also true when $k=j:\left\langle\varphi_{j}^{\prime}, \varphi_{j}\right\rangle=\int_{0}^{+\infty} \varphi_{j}^{\prime}(x) \varphi_{j}(x) d x=-(1 / 2) \varphi_{j}^{2}(0)=$ $-2 / 2=-1$. Thus we obtain:

$$
\begin{aligned}
\varphi_{j}^{\prime}(x) & =\sum_{k=0}^{j} a_{k}^{(j)} \varphi_{k}(x)=-2 \sum_{k=0}^{j} \varphi_{k}(x)-\sum_{k=0}^{j}\left\langle\varphi_{j}, \varphi_{k}^{\prime}\right\rangle \varphi_{k}(x) \\
& =-\varphi_{j}(x)-2 \sum_{k=0}^{j-1} \varphi_{k}(x)-\sum_{k=0}^{j-1}\left\langle\varphi_{j}, \varphi_{k}^{\prime}\right\rangle \varphi_{k}(x)
\end{aligned}
$$

Note that the $\left\langle\varphi_{j}, \varphi_{k}^{\prime}\right\rangle$ are zero for $k \leq j-1$. Thus we obtain the first formula. The bound on $\varphi_{j}^{\prime}(x)$ follows from $\left|\varphi_{j}(x)\right| \leq \sqrt{2}$ and the bound on $\left|x \varphi_{j}^{\prime}(x)+\varphi_{j}(x)\right|$ from (47).

7.1.2. Formulae for $\delta>-1$. The following holds:

implying

$$
\begin{gathered}
x L_{j}^{(\delta)}(x)=-(j+1) L_{j+1}^{(\delta)}(x)+(2 j+\delta+1) L_{j}^{(\delta)}(x)-(j+\delta) L_{j-1}^{(\delta)}(x), \\
x\left(L_{j}^{(\delta)}\right)^{\prime}(x)=j L_{j}^{(\delta)}(x)-(j+\delta) L_{j-1}^{(\delta)}(x),
\end{gathered}
$$

$$
\begin{gathered}
x \varphi_{j}^{(\delta)}(x)=-\frac{\sqrt{(j+1)(j+\delta+1)}}{2} \varphi_{j+1}^{(\delta)}(x)+\left(j+\frac{\delta+1}{2}\right) \varphi_{j}^{(\delta)}(x)-\frac{\sqrt{j(j+\delta)}}{2} \varphi_{j-1}^{(\delta)}(x), \\
\left(x \varphi_{j}^{(\delta)}(x)\right)^{\prime}=-\frac{\sqrt{j(j+\delta)}}{2} \varphi_{j-1}^{(\delta)}(x)+\frac{1}{2} \varphi_{j}^{(\delta)}(x)+\frac{\sqrt{(j+1)(j+\delta+1)}}{2} \varphi_{j+1}^{(\delta)}(x) .
\end{gathered}
$$

7.1.3. Asymptotic formulae. From Askey and Wainger (1965), we have for $\nu=4 k+2 \delta+2$, and $k$ large enough

$$
\left|\varphi_{k}^{(\delta)}(x / 2)\right| \leq C\left\{\begin{array}{lll}
a) & (x \nu)^{\delta / 2} & \text { if } 0 \leq x \leq 1 / \nu \\
b) & (x \nu)^{-1 / 4} & \text { if } 1 / \nu \leq x \leq \nu / 2 \\
c) & \nu^{-1 / 4}(\nu-x)^{-1 / 4} & \text { if } \nu / 2 \leq x \leq \nu-\nu^{1 / 3} \\
d) & \nu^{-1 / 3} & \text { if } \nu-\nu^{1 / 3} \leq x \leq \nu+\nu^{1 / 3} \\
e) & \nu^{-1 / 4}(x-\nu)^{-1 / 4} e^{-\gamma_{1} \nu^{-1 / 2}(x-\nu)^{3 / 2}} & \text { if } \nu+\nu^{1 / 3} \leq x \leq 3 \nu / 2 \\
f) & e^{-\gamma_{2} x} & \text { if } x \geq 3 \nu / 2
\end{array}\right.
$$

where $\gamma_{1}$ and $\gamma_{2}$ are positive and fixed constants. From these estimates, we can prove

Lemma 7.2. Let $p$ be a nonnegative real number. Assume that a random variable $R$ has density $f_{R}$ on $\mathbb{R}^{+}$and that $\mathbb{E}\left(R^{p-1 / 2}\right)<+\infty$ and $\mathbb{E}\left(R^{p}\right)<+\infty$. For $k$ large enough,

$$
\int_{0}^{+\infty} x^{p}\left[\varphi_{k}^{(\delta)}(x)\right]^{2} f_{R}(x) d x \leq \frac{c}{\sqrt{k}}
$$

where $c=c_{p}>0$ is a constant depending on $p$ and $\mathbb{E}\left(R^{p-1 / 2}\right), \mathbb{E}\left(R^{p}\right)$.

Proof of Lemma 7.2. We have six terms to compute to find the order of

$$
\int_{0}^{+\infty} x^{p}\left[\varphi_{k}^{(\delta)}(x)\right]^{2} f_{R}(x) d x=\left(1 / 2^{p+1}\right) \int_{0}^{+\infty} u^{p}\left[\varphi_{k}^{(\delta)}(u / 2)\right]^{2} f_{R}(u / 2) d u:=\sum_{\ell=1}^{6} I_{\ell} .
$$

a) $I_{1} \lesssim \frac{1}{2^{p+1}} \int_{0}^{1 / \nu} u^{p}(u \nu)^{\delta} f_{R}(u / 2) d u \lesssim\left\|f_{R}\right\| \nu^{-(p+1 / 2)} \lesssim\left\|f_{R}\right\| k^{-(p+1 / 2)}$

b) $I_{2} \lesssim \nu^{-1 / 2} \int_{1 / \nu}^{\nu / 2} f_{R}(u / 2) u^{p-1 / 2} d u \lesssim k^{-1 / 2} \mathbb{E}\left(R^{p-1 / 2}\right)$. 

c) $I_{3} \lesssim \nu^{-1 / 2} \nu^{-1 / 6} \int_{\nu / 2}^{\nu-\nu^{1 / 3}} u^{p} f_{R}(u / 2) d u=o(1 / \sqrt{k})$, as $\nu-u \geq \nu^{1 / 3}$ and $\mathbb{E}\left(R^{p}\right)<+\infty$.
d) $I_{4} \lesssim \nu^{-2 / 3} \int_{\nu-\nu^{1 / 3}}^{\nu+\nu^{1 / 3}} u^{p} f_{R}(u / 2) d u=o(1 / \sqrt{k})$ as $\mathbb{E}\left(R^{p}\right)<+\infty$.
e) $I_{5} \lesssim \nu^{-1 / 2} \int_{\nu+\nu^{1 / 3}}^{3 \nu / 2} u^{p}(u-\nu)^{-1 / 2} f_{R}(u / 2) d u \lesssim \nu^{-1 / 2} \nu^{-1 / 6} \mathbb{E}\left(R^{p}\right)=o(1 / \sqrt{k})$,

(exp is bounded by $1, u-\nu \geq \nu^{1 / 3}$ ).

f) $I_{6} \lesssim e^{-\gamma_{2}(3 \nu / 2)} \mathbb{E}\left(R^{p}\right)=o(1 / \sqrt{k})$.

The result of Lemma 7.2 follows from these orders.

7.2. Formulae for Hermite functions. Using (see Abramowitz and Stegun (1964))

$$
2 x H_{j}(x)=H_{j+1}(x)+2 j H_{j-1}(x), \quad H_{j}^{\prime}(x)=2 j H_{j-1}(x), \quad j \geq 1 .
$$

we get:

(52) $\sqrt{2} h_{j}^{\prime}(x)=\sqrt{j} h_{j-1}(x)-\sqrt{j+1} h_{j+1}(x), 2 x h_{j}(x)=\sqrt{2(j+1)} h_{j+1}(x)+\sqrt{2 j} h_{j-1}(x)$.

Moreover, $h_{j}^{*}(t)=\sqrt{2 \pi} i^{j} h_{j}(t)$.

Lemma 7.3. There exist constants $C_{\infty}^{\prime}, C^{\prime \prime}{ }_{\infty}$ such that, for all $j \geq 0$,

(1) $\left\|h_{j}^{\prime}\right\|_{\infty} \leq C_{\infty}^{\prime}(j+1)^{5 / 12}$

(2) $\left\|y h_{j}^{\prime}+h_{j}\right\|_{\infty} \leq C^{\prime \prime}{ }_{\infty}(j+1)^{11 / 12}$.

Proof of Lemma 7.3. For (1), we use (52) and the first bound:

$$
\left\|h_{j}^{\prime}\right\|_{\infty} \leq \frac{C_{\infty}}{\sqrt{2}}\left(1+\frac{\sqrt{j+1}}{(j+2)^{1 / 12}}\right) \leq \sqrt{2} C_{\infty}(j+1)^{5 / 12} .
$$

Next, (52) implies that

$$
\begin{aligned}
\left(y h_{j}(y)\right)^{\prime} & =\sqrt{(j+1) / 2} h_{j+1}^{\prime}(y)+\sqrt{j / 2} h_{j-1}^{\prime}(y) \\
& =\frac{1}{2}\left(\sqrt{j(j-1)} h_{j-2}(y)+h_{j}(y)-\sqrt{(j+1)(j+2)} h_{j+2}(y)\right),
\end{aligned}
$$

where the last line follows from (52). Thus, $\left|y h_{j}^{\prime}(y)+h_{j}(y)\right| \leq 2 C_{\infty}(j+1)^{11 / 12}$. So we get (2).

The Hermite polynomials are linked with the Laguerre polynomials as follows (see Abramowitz and Stegun, 1964, p.779, 22.5.40, 22.5.41). For $x \geq 0$,

$$
H_{2 n}(x)=(-1)^{n} 2^{2 n} n ! L_{n}^{(-1 / 2)}\left(x^{2}\right), \quad H_{2 n+1}(x)=(-1)^{n} 2^{2 n+1} n ! x L_{n}^{(1 / 2)}\left(x^{2}\right) .
$$

The Hermite polynomials are even for even $n$ and odd for odd $n$. From this, we deduce the following link between Hermite and Laguerre functions:

Lemma 7.4. For $x \geq 0$,

$$
h_{2 n}(x)=(-1)^{n} \sqrt{x / 2} \varphi_{n}^{(-1 / 2)}\left(x^{2} / 2\right), \quad h_{2 n+1}(x)=(-1)^{n} \sqrt{x / 2} \varphi_{n}^{(1 / 2)}\left(x^{2} / 2\right) .
$$

Proof of Lemma 7.4. We have

$$
h_{2 n}(x)=c_{2 n} e^{-x^{2} / 2} H_{2 n}(x)=(-1)^{n} d_{2 n} L_{n}^{(-1 / 2)}\left(x^{2}\right) e^{-x^{2} / 2}\left(x^{2}\right)^{-1 / 4}\left(x^{2}\right)^{1 / 4},
$$


with $c_{2 n}, d_{2 n}>0$. Thus, $h_{2 n}(x)=(-1)^{n} d_{2 n} \phi_{n}^{(-1 / 2)}\left(x^{2}\right) \sqrt{x}=(-1)^{n} d_{2 n} \sqrt{x / 2} \varphi_{n}^{(-1 / 2)}\left(x^{2} / 2\right)$. Therefore,

$$
\int_{0}^{\infty} h_{2 n}^{2}(x) d x=1 / 2=d_{2 n}^{2} \int_{0}^{\infty}\left(\phi_{n}^{(-1 / 2)}\left(x^{2}\right)\right)^{2} x d x=d_{n}^{2} / 2 .
$$

This shows $d_{2 n}^{2}=1$, hence $d_{2 n}=1$. Analogously,

$$
h_{2 n+1}(x)=c_{2 n+1} e^{-x^{2} / 2} H_{2 n+1}(x)=(-1)^{n} d_{2 n+1} \sqrt{x} L_{n}^{(1 / 2)}\left(x^{2}\right) e^{-x^{2} / 2} \sqrt{x},
$$

with $c_{2 n+1}, d_{2 n+1}>0$. We get $h_{2 n+1}(x)=(-1)^{n} d_{2 n+1} \phi_{n}^{(1 / 2)}\left(x^{2}\right) \sqrt{x}$. We conclude as above that $d_{2 n+1}=1$.

7.3. Asymptotic formulae. Now, we can use the estimates of Askey and Wainger (1965) for $\varphi_{n}^{(\delta)}$ to obtain the following result:

Lemma 7.5. Let $p$ be a nonnegative real number. Assume that a random variable $R$ has density $f_{R}$ on $\mathbb{R}$ and that $\mathbb{E}\left(|R|^{p+2 / 3}\right)<+\infty$. For $k$ large enough,

$$
\int_{0}^{+\infty} x^{p} h_{k}^{2}(x) f_{R}(x) d x \leq \frac{c}{\sqrt{k}}
$$

where $c>0$ is a constant which depends on $p$.

Proof of Lemma 7.5. We start with the even indexes. Again,we have six terms to compute to find the order of

$$
\int_{0}^{+\infty} x^{p} h_{2 k}^{2}(x) f_{R}(x) d x=(1 / 2) \int_{0}^{+\infty} x^{p+1}\left(\varphi_{n}^{(-1 / 2)}\left(x^{2} / 2\right)\right)^{2} f_{R}(x) d x:=\sum_{\ell=1}^{6} J_{\ell} .
$$

We take $\nu=4 k+1$.

$$
\begin{aligned}
J_{1} & =\int_{0}^{1 / \sqrt{\nu}} x^{p} h_{2 k}^{2}(x) f_{R}(x) d x=\frac{1}{2} \int_{0}^{1 / \sqrt{\nu}} x^{p+1}\left(\varphi_{n}^{(-1 / 2)}\left(x^{2} / 2\right)\right)^{2} f_{R}(x) d x \\
& \leq C \int_{0}^{1 / \sqrt{\nu}} x^{p+1}\left(\left(x^{2} \nu\right)^{-1 / 4}\right)^{2} f_{R}(x) d x \leq \frac{C}{\nu^{(p+1) / 2}} \int_{0}^{1 / \sqrt{\nu}} f_{R}(x) d x \leq \frac{C}{\nu^{(p+1) / 2}}, \\
J_{2} & =\int_{1 / \sqrt{\nu}}^{\sqrt{\nu / 2}} x^{p} h_{2 k}^{2}(x) f_{R}(x) d x \leq \frac{C}{2} \int_{1 / \sqrt{\nu}}^{\sqrt{\nu / 2}} x^{p+1}\left(x^{2} \nu\right)^{-1 / 2} f_{R}(x) d x \leq \frac{C}{2 \sqrt{\nu}} \mathbb{E}\left(|R|^{p}\right), \\
J_{3} & =\int_{\sqrt{\nu / 2}}^{\left(\nu-\nu^{1 / 3}\right)^{1 / 2}} x^{p} h_{2 k}^{2}(x) f_{R}(x) d x \\
& \leq \frac{C}{2} \int_{\sqrt{\nu / 2}}^{\left(\nu-\nu^{1 / 3}\right)^{1 / 2}} x^{1 / 3} x^{p+2 / 3} \nu^{-1 / 2}\left(\nu-x^{2}\right)^{-1 / 2} f_{R}(x) d x \leq \frac{C}{2 \sqrt{\nu}} \mathbb{E}\left(|R|^{p+2 / 3}\right), \\
J_{4} & =\int_{\left(\nu-\nu^{1 / 3}\right)^{1 / 2}}^{\left(\nu+\nu^{1 / 3}\right)^{1 / 2}} x^{p} h_{2 k}^{2}(x) f_{R}(x) d x \leq \frac{C}{2} \nu^{-1 / 2} \mathbb{E}\left(|R|^{p+2 / 3}\right), \\
J_{5} & =\int_{\left(\nu+\nu^{1 / 3}\right)^{1 / 2}}^{\sqrt{3 \nu / 2}} x^{p} h_{2 k}^{2}(x) f_{R}(x) d x \leq \frac{C}{2 \sqrt{\nu}} \mathbb{E}\left(|R|^{p+2 / 3}\right), \\
J_{6} & =\int_{\sqrt{3 \nu / 2}}^{+\infty} x^{p} h_{2 k}^{2}(x) f_{R}(x) d x \leq C^{\prime} \exp \left(-3 \gamma_{2} \nu / 2\right) .
\end{aligned}
$$


Now, we deal with

$$
\int_{0}^{+\infty} x^{p} h_{2 k+1}^{2}(x) f_{R}(x) d x=(1 / 2) \int_{0}^{+\infty} x^{p+1}\left(\varphi_{n}^{(1 / 2)}\left(x^{2} / 2\right)\right)^{2} f_{R}(x) d x:=\sum_{\ell=1}^{6} K_{\ell},
$$

and take $\nu=4 k+3$. The only difference is on the first term:

$$
K_{1}=\int_{0}^{1 / \sqrt{\nu}} x^{p} h_{2 k+1}^{2}(x) f_{R}(x) d x \leq \frac{C}{2} \int_{0}^{1 / \sqrt{\nu}} x^{p}\left(x^{2} \nu\right)^{1 / 2} f_{R}(x) d x \leq \frac{C}{2 \nu^{(p+1) / 2}} .
$$

This ends the proof.

7.4. Proof of Proposition 3.1. We first prove the result in the Laguerre case. From Lemma 7.2 with $\delta=0, p=0$, we get that, if $\mathbb{E}\left(1 / \sqrt{X_{1}}\right)<+\infty$, then for $j$ large enough,

$$
\mathbb{E}\left(\varphi_{j}^{2}\left(X_{1}\right)\right)=\int_{0}^{+\infty} \varphi_{j}^{2}(x) f(x) d x \leq \frac{c}{\sqrt{j}},
$$

where $c>0$ is a constant. As a consequence, for $m$ large enough,

$$
\mathbb{E}\left(\left\|\hat{f}_{m}^{X}-f_{m}\right\|^{2}\right) \leq \sum_{j=0}^{m-1} \mathbb{E}\left[\varphi_{j}^{2}\left(X_{1}\right)\right] \lesssim m^{1 / 2},
$$

which gives the announced result.

In the Hermite case, we apply Lemma 7.5 for $p=0$. Thus for $k$ large enough, $V_{m}^{X} \lesssim$ $\Phi_{0}^{2}+\sum_{k=1}^{m-1} k^{-1 / 2}=O(\sqrt{m})$, and the result follows.

7.5. Proof of Proposition 3.2. We only study the Laguerre case, as the Hermite case is proved in Belomestny et al. (2017), Proposition 2.2. By Theorem 8.22.5 in Szego (1959), reminding formula (44), we have: for all $\delta>-1$, and for $\underline{b} / k \leq x \leq \bar{b}$, where $\underline{b}, \bar{b}$ are arbitrary constants,

$$
\varphi_{k}^{(\delta)}(x)=c(k x)^{-1 / 4}\left(\cos (2 \sqrt{2} \sqrt{k x}-\delta \pi / 2-\pi / 4)+(k x)^{-1 / 2} O(1)\right),
$$

where $O(1)$ is uniform on $[\underline{b} / k, \bar{b}]$ and $c=2^{1 / 4} / \sqrt{\pi}$.

Take $k$ such that $\underline{b} / k<a<b<\bar{b}$ and set $d=\inf _{a \leq x \leq b} f(x)$. Then write

$$
\int_{0}^{+\infty} \varphi_{k}^{2}(x) f(x) d x \geq d \int_{a}^{b} \varphi_{k}^{2}(x) d x
$$

We have,

$$
\varphi_{k}^{2}(x)=\frac{c^{2}}{2}(k x)^{-1 / 2}(1+\sin (4 \sqrt{2 k x}))+(k x)^{-1} O(1) .
$$

Therefore, as $\int_{a}^{b} \sin (4 \sqrt{2 k x}) d x=2 \int_{\sqrt{a}}^{\sqrt{b}} \sin (4 \sqrt{2 k} u) u d u=O(1 / \sqrt{k})$,

$$
\int_{a}^{b} \varphi_{k}^{2}(x) d x \geq \frac{c^{2}}{2 \sqrt{a}} k^{-1 / 2}\left(b-a+O\left(\frac{1}{\sqrt{k}}\right)\right)+O\left(\frac{1}{k}\right) .
$$

Consequently, for $k$ large enough, we get

$$
\int_{0}^{+\infty} \varphi_{k}^{2}(x) f(x) d x \geq c^{\prime} / \sqrt{k} .
$$


7.6. Proof of Proposition 3.3. Let $X$ have density $f(x)=\int_{0}^{\infty} \theta \exp (-\theta x) d \Pi(\theta)$. Then, $X={ }_{\mathcal{L}} Z / T$ where $Z, T$ are independent, $Z$ has exponential distribution with parameter 1 and $T$ has distribution $\Pi$. For fixed $\theta>0$, elementary computations yield

$$
\mathbb{E} \varphi_{j}(Z / \theta)=\int_{0}^{+\infty} \theta \exp (-\theta x) \varphi_{j}(x) d x=\sqrt{2} \frac{\theta}{\theta+1}\left(\frac{\theta-1}{\theta+1}\right)^{j} .
$$

Then, we compute

$$
\mathbb{E} \varphi_{j}(X)=\mathbb{E}\left(\mathbb{E}\left(\varphi_{j}(Z / T) \mid T\right)\right)=\int_{0}^{+\infty} \sqrt{2} \frac{\theta}{\theta+1}\left(\frac{\theta-1}{\theta+1}\right)^{j} d \Pi(\theta)=\mathbb{E}\left(\sqrt{2} \frac{T}{T+1}\left(\frac{T-1}{T+1}\right)^{j}\right)
$$

Consequently,

$$
\sum_{j \geq m} a_{j}^{2}(f)=\sum_{j \geq m} 2\left[\mathbb{E} \frac{T}{T+1}\left(\frac{T-1}{T+1}\right)^{j}\right]^{2} \leq \frac{1}{2} \mathbb{E} T\left(\frac{T-1}{T+1}\right)^{2 m} \leq \frac{v}{2} \rho^{2 m} .
$$

Note that for $Z \sim \mathcal{E}(1)$

$$
\mathbb{E}\left(\frac{1}{\sqrt{X}}\right) \leq \sqrt{v} \mathbb{E}\left(\frac{1}{\sqrt{Z}}\right)<+\infty
$$

Choosing $m_{\mathrm{opt}}=[\log (n) /|\log (\rho)|]$ and using Proposition 3.1, the result follows. $\square$

7.7. Proof of Theorem 3.1. The Hermite case is proved in Theorem 2.1 of Belomestny et al. (2017b).

The Laguerre case is identical, except for one point. We use that $\sup _{x} \sum_{j=0}^{m-1} \varphi_{j}^{2}(x) \leq 2 m$, instead of $\sup _{x} \sum_{j=0}^{m-1} h_{j}^{2}(x) \leq C_{\infty}^{\prime} m^{5 / 6}$. This explains the new bound on $m_{n}$.

7.8. Proof of formula (13)-(14) and Lemma 4.1. Equality (13) is elementary. For $y \geq 0$,

$$
\begin{aligned}
\bar{F}_{Y}(y) & =\int_{y}^{+\infty} f_{Y}(z) d z=\int_{y}^{+\infty} \int_{z}^{+\infty} \frac{f(x)}{x} d x d z=\int\left(\int_{y}^{x} d z\right) \frac{f(x)}{x} \mathbf{I}(y \leq x) d x \\
& =\int_{y}^{+\infty}(x-y) \frac{f(x)}{x} d x=\int_{y}^{+\infty} f(x) d x-y \int_{y}^{+\infty} \frac{f(x)}{x} d x=\bar{F}(y)-y f_{Y}(y) .
\end{aligned}
$$

For $y \leq 0$,

$$
\begin{aligned}
F_{Y}(y) & =\int_{-\infty}^{y} f_{Y}(z) d z=\int_{-\infty}^{y} d z \int_{-\infty}^{z} \frac{f(x)}{|x|} d x=\int\left(\int_{x}^{y} d z\right) \frac{f(x)}{|x|} \mathbb{I}(x \leq y) d x \\
& =\int_{-\infty}^{y}(y-x) \frac{f(x)}{|x|} d x=y f_{Y}(y)+F(y)
\end{aligned}
$$

Thus, $\bar{F}_{Y}(y)=\bar{F}(y)-y f_{Y}(y)$, which is (14).

For $(15)$, by (13), $y f_{Y}(y)$ tends to 0 as both $y$ tends to $+\infty$ and $-\infty$. Integrating by parts yields

$$
\begin{aligned}
& \int_{\mathbb{R}} f_{Y}(y)\left(t(y)+y t^{\prime}(y)\right) d y=-\int_{\mathbb{R}} y t(y)\left(f_{Y}(y)\right)^{\prime} d y \\
= & -\left[\int_{0}^{+\infty} y t(y)\left(-\frac{f(y)}{y}\right) d y+\int_{-\infty}^{0} y t(y) \frac{f(y)}{|y|} d y\right]=\int_{-\infty}^{+\infty} t(y) f(y) d y .
\end{aligned}
$$


Note that $\mathbb{E} Y_{1}^{2} t^{2}\left(Y_{1}\right) \leq \mathbb{E} X_{1}^{2} t^{2}\left(U_{1} X_{1}\right)$. Then,

$$
\begin{aligned}
\mathbb{E} X^{2} t^{2}\left(U_{1} X_{1}\right) & =\int_{0}^{+\infty} x f(x)\left(\int_{0}^{x} t^{2}(v) d v\right) d x+\int_{-\infty}^{0}|x| f(x)\left(\int_{x}^{0} t^{2}(v) d v\right) d x \\
& \leq \mathbb{E}\left(\left|X_{1}\right|\right)\|t\|^{2} .
\end{aligned}
$$

\subsection{Proof of Proposition 4.1.}

- Laguerre case. First, we bound $V_{m}$ in the case $\psi_{j}=\varphi_{j}$. We write

$$
\mathbb{E}\left[\left(Y_{1} \varphi_{j}^{\prime}\left(Y_{1}\right)+\varphi_{j}\left(Y_{1}\right)\right)^{2}\right]=\mathbb{E}\left[\left(Y_{1} \varphi_{j}^{\prime}\left(Y_{1}\right)\right)^{2}\right]+\mathbb{E}\left[2 Y_{1} \varphi_{j}^{\prime}\left(Y_{1}\right) \varphi_{j}\left(Y_{1}\right)+\varphi_{j}^{2}\left(Y_{1}\right)\right] .
$$

Formula (15) applied to $t=\varphi_{j}^{2}$ yields

$$
\mathbb{E}\left[2 Y_{1} \varphi_{j}^{\prime}\left(Y_{1}\right) \varphi_{j}\left(Y_{1}\right)+\varphi_{j}^{2}\left(Y_{1}\right)\right]=\mathbb{E}\left(\varphi_{j}^{2}\left(X_{1}\right)\right) .
$$

Therefore

$$
\mathbb{E}\left[\left(Y_{1} \varphi_{j}^{\prime}\left(Y_{1}\right)+\varphi_{j}\left(Y_{1}\right)\right)^{2}\right]=\mathbb{E}\left[\left(Y_{1} \varphi_{j}^{\prime}\left(Y_{1}\right)\right)^{2}\right]+\mathbb{E}\left(\varphi_{j}^{2}\left(X_{1}\right)\right)
$$

where the second rhs term is the same as in the direct case, and thus $\mathbb{E}\left(\varphi_{j}^{2}\left(X_{1}\right)\right) \lesssim j^{-1 / 2}$ for $j$ large enough and if $\mathbb{E}\left(1 / \sqrt{X_{1}}\right)<+\infty$.

For the first rhs term, we use formula (48): $y \varphi_{j}^{\prime}(y)=-\sqrt{2 j y} \varphi_{j-1}^{(1)}(y)-y \varphi_{j}(y)$. Therefore, for $j$ large enough, we have

$$
\int_{0}^{+\infty}\left[y \varphi_{j}^{\prime}(y)\right]^{2} f_{Y}(y) d y \lesssim j \int_{0}^{+\infty} y\left[\varphi_{j-1}^{(1)}(y)\right]^{2} f_{Y}(y) d y+\int_{0}^{+\infty} y^{2} \varphi_{j}^{2}(y) f_{Y}(y) d y .
$$

Now we apply Lemma 7.2 to the first term with $p=1$ and $\delta=1$, so that we get that $\int_{0}^{+\infty} y\left[\varphi_{j-1}^{(1)}(y)\right]^{2} f_{Y}(y) d y \lesssim 1 / \sqrt{j}$ if $\mathbb{E}\left(X_{1}\right)$ is finite.

For the second term, we use formula (46) and $u v \leq\left(u^{2}+v^{2}\right) / 2$ and obtain

$$
\begin{aligned}
\int_{0}^{+\infty} y^{2} \varphi_{j}^{2}(y) f_{Y}(y) d y= & \int_{0}^{+\infty} y \varphi_{j}(y)\left[-\frac{j+1}{2} \varphi_{j+1}(y)+\left(j+\frac{1}{2}\right) \varphi_{j}(y)-\frac{j}{2} \varphi_{j-1}(y)\right] f_{Y}(y) d y \\
\leq & \left(\frac{3 j}{2}+\frac{3}{4}\right) \int_{0}^{+\infty} y \varphi_{j}^{2}(y) f_{Y}(y) d y+\frac{j+1}{4} \int_{0}^{+\infty} y \varphi_{j+1}^{2}(y) f_{Y}(y) d y \\
& +\frac{j}{4} \int_{0}^{+\infty} y \varphi_{j-1}^{2}(y) f_{Y}(y) d y=O(\sqrt{j})
\end{aligned}
$$

by Lemma 7.2 with $p=1$ and $\delta=0$, if $\mathbb{E}\left(X_{1}\right)$ is finite.

Therefore, we get that, for $m$ large enough, if $\mathbb{E}\left(1 / \sqrt{X_{1}}\right)<+\infty$ and $\mathbb{E}\left(X_{1}\right)<+\infty$,

$$
V_{m}=\sum_{j=0}^{m-1} \mathbb{E}\left[\left(Y_{1} \varphi_{j}^{\prime}\left(Y_{1}\right)+\varphi_{j}\left(Y_{1}\right)\right)^{2}\right] \lesssim m^{3 / 2}
$$

- Hermite case. Now, we bound $V_{m}$ in the case $\psi_{j}=h_{j}$. Using (52), we get that

$$
x h_{j}^{\prime}(x)=\frac{x}{\sqrt{2}}\left(\sqrt{j} h_{j-1}(x)-\sqrt{j+1} h_{j+1}(x)\right)
$$

and the order of $\int_{\mathbb{R}}\left(y h_{j}^{\prime}(y)+h_{j}(y)\right)^{2} f_{Y}(y) d y$ follows from Lemma 7.5 applied for $p=2$ and $p=0$. We obtain a bound of order $\sqrt{j}$ for $j$ large enough, if $\mathbb{E}\left(\left|X_{1}\right|^{2+2 / 3}\right)<+\infty$. Therefore, we get that, for $m$ large enough, if $\mathbb{E}\left(\left|X_{1}\right|^{2+2 / 3}\right)<+\infty$,

$$
V_{m}=\sum_{j=0}^{m-1} \mathbb{E}\left[\left(Y_{1} h_{j}^{\prime}\left(Y_{1}\right)+h_{j}\left(Y_{1}\right)\right)^{2}\right] \lesssim m^{3 / 2}
$$


7.10. Proof of Remark 4.1. Using Formula (15) with $t=h_{j}^{2}$,

$$
V_{m}=\sum_{j=0}^{m-1} \mathbb{E}\left(Y_{1}^{2}\left(h_{j}^{\prime}\left(Y_{1}\right)\right)^{2}\right)+\sum_{j=0}^{m-1} \mathbb{E} h_{j}^{2}\left(X_{1}\right)=\sum_{j=0}^{m-1} \mathbb{E}\left(Y_{1}^{2}\left(h_{j}^{\prime}\left(Y_{1}\right)\right)^{2}\right)+V_{m}^{X} .
$$

We know that $V_{m}^{X} \leq \mathrm{cm}^{5 / 6}$. For the first term of the r.h.s. above, we can use Lemma 4.1:

$$
\mathbb{E}\left(Y_{1}^{2}\left(h_{j}^{\prime}\left(Y_{1}\right)\right)^{2}\right) \leq \mathbb{E}\left|X_{1}\right|\left\|h_{j}^{\prime}\right\|^{2}=\mathbb{E}\left|X_{1}\right|\left(j+\frac{1}{2}\right)
$$

as $h_{j}^{\prime}=\left(\sqrt{j} h_{j-1}-\sqrt{j+1} h_{j+1}\right) / \sqrt{2}($ see $(52))$. Consequently,

$$
\sum_{j=0}^{m-1} \mathbb{E}\left(Y_{1}^{2}\left(h_{j}^{\prime}\left(Y_{1}\right)\right)^{2}\right) \leq \frac{m^{2}}{2} \mathbb{E}\left|X_{1}\right| .
$$

Joining (55) and (56) gives the first risk bound.

For the second point, we note that

$$
\mathbb{E}\left(Y_{1}^{2}\left(h_{j}^{\prime}\left(Y_{1}\right)\right)^{2}\right) \leq \mathbb{E} Y^{2}\left\|h_{j}^{\prime}\right\|_{\infty}^{2}=\frac{1}{3} \mathbb{E} X_{1}^{2}\left\|h_{j}^{\prime}\right\|_{\infty}^{2}
$$

where $\left\|h_{j}^{\prime}\right\|_{\infty} \leq C_{\infty}^{\prime}(j+1)^{5 / 12}$ by Lemma 7.3. Therefore,

$$
\sum_{j=0}^{m-1} \mathbb{E}\left(Y_{1}^{2}\left(h_{j}^{\prime}\left(Y_{1}\right)\right)^{2}\right) \leq \frac{\left(C_{\infty}^{\prime}\right)^{2}}{3} \mathbb{E} X_{1}^{2} m^{11 / 6} .
$$

This gives the second risk bound.

\subsection{Proof of Proposition 4.3.}

- Laguerre case. By assumption, there exist $0<a<b$ and $c>0$ such that $\inf _{a \leq x \leq b} f(x)>$ $c>0$. This implies that we can find $0<a^{\prime}<b^{\prime}$ and $c^{\prime}$ such that $\inf _{a^{\prime} \leq y \leq b^{\prime}} y f_{Y}(y)=c^{\prime}>0$. Indeed,

$$
\inf _{a / 2 \leq y \leq a} y f_{Y}(y) \geq \frac{a}{2} \int_{a}^{b} \frac{f(x)}{x} d x \geq \frac{c a}{2} \log (b / a) .
$$

Thus $\inf _{a^{\prime} \leq y \leq b^{\prime}} y f_{Y}(y)=c^{\prime}>0$ holds with $a^{\prime}=a / 2, b^{\prime}=a, c^{\prime}=(c a / 2) \log (b / a)$.

Now

$$
\begin{aligned}
& \mathbb{E}\left[\left(Y_{1} \varphi_{j}^{\prime}\left(Y_{1}\right)+\varphi_{j}\left(Y_{1}\right)\right)^{2}\right]=\mathbb{E}\left[\left(Y_{1} \varphi_{j}^{\prime}\left(Y_{1}\right)\right)^{2}\right]+\mathbb{E}\left(\varphi_{j}^{2}\left(X_{1}\right)\right) \\
& \geq \mathbb{E}\left\{\left[\sqrt{2 j Y_{1}} \varphi_{j-1}^{(1)}\left(Y_{1}\right)+Y_{1} \varphi_{j}\left(Y_{1}\right)\right]^{2}\right\} \\
& \geq 2 j \int_{a^{\prime}}^{b^{\prime}} y\left[\varphi_{j-1}^{(1)}(y)\right]^{2} f_{Y}(y) d y+2 \sqrt{2 j} \int_{a^{\prime}}^{b^{\prime}} y \sqrt{y} \varphi_{j-1}^{(1)}(y) \varphi_{j}(y) f_{Y}(y) d y \\
&:=T_{1}+T_{2} .
\end{aligned}
$$

We have, by applying Lemma 7.2 for $p=0$ if $\mathbb{E}\left(1 / \sqrt{X_{1}}\right)<+\infty$,

$$
\left|T_{2}\right| \leq \sqrt{2 j}\left(b^{\prime}\right)^{3 / 2} \int_{a^{\prime}}^{b^{\prime}}\left(\left[\varphi_{j-1}^{(1)}\right]^{2}(y)+\varphi_{j}^{2}(y)\right) f_{Y}(y) d y=O(1) .
$$

Next, using (54), we proceed as in the proof of Proposition 3.2,

$$
T_{1} \geq 2 j c^{\prime} \int_{a^{\prime}}^{b^{\prime}}\left[\varphi_{j-1}^{(1)}\right]^{2}(y) d y \geq c \sqrt{j}
$$


We conclude that $V_{m} \geq C m^{3 / 2}+O(m)$.

- Hermite case. Analogously, we can find $a^{\prime}<b^{\prime}$ and $c^{\prime}$ such that $\inf _{a^{\prime} \leq y \leq b^{\prime}} y^{2} f_{Y}(y)=c^{\prime}>0$. For instance if $b>0$, we can assume $a>0$. Then

$$
\inf _{a / 2 \leq y \leq a} y^{2} f_{Y}(y) \geq \frac{a^{2}}{4} \int_{a}^{b} \frac{f(x)}{x} d x \geq \frac{c a^{2}}{4} \log (b / a) .
$$

Thus $\inf _{a^{\prime} \leq y \leq b^{\prime}} y^{2} f_{Y}(y)=c^{\prime}>0$ holds with $a^{\prime}=a / 2, b^{\prime}=a, c^{\prime}=\left(c a^{2} / 4\right) \log (b / a)$.

Here, we can use as in Walter (1977), the following expression for the Hermite function $h_{j}$ (see Szegö (1959, p.248)):

$$
h_{j}(x)=\lambda_{j} \cos \left((2 j+1)^{1 / 2} x-\frac{j \pi}{2}\right)+\frac{1}{(2 j+1)^{1 / 2}} \xi_{j}(x)
$$

where $\lambda_{j}=\left|h_{j}(0)\right|$ if $j$ is even, $\lambda_{j}=\left|h_{j}^{\prime}(0)\right| /(2 j+1)^{1 / 2}$ if $j$ is odd and

$$
\xi_{j}(x)=\int_{0}^{x} \sin \left[(2 j+1)^{1 / 2}(x-t)\right] t^{2} h_{j}(t) d t .
$$

We have

$$
\lambda_{2 j}=\frac{(2 j) !^{1 / 2}}{2^{j} j ! \pi^{1 / 4}}, \quad \lambda_{2 j+1}=\lambda_{2 j} \frac{\sqrt{2 j+1}}{\sqrt{2 j+3 / 2}} .
$$

By the Stirling formula and its proof, $\lambda_{2 j} \sim \pi^{-1 / 2} j^{-1 / 4}, \lambda_{2 j+1} \sim \pi^{-1 / 2} j^{-1 / 4}$ and for all $j$, there exists constants $c_{1}, c_{2}$ such that, for all $j \geq 1$,

$$
\frac{c_{1}}{\pi^{1 / 2} j^{1 / 4}} \leq \lambda_{j} \leq \frac{c_{2}}{\pi^{1 / 2} j^{1 / 4}}
$$

By derivating (58), we get

$$
h_{j}^{\prime}(x)=-\sqrt{2 j+1} \lambda_{j} \sin \left((2 j+1)^{1 / 2} x-\frac{j \pi}{2}\right)+\frac{1}{(2 j+1)^{1 / 2}} \xi_{j}^{\prime}(x)
$$

with

$$
\xi_{j}^{\prime}(x)=\sqrt{2 j+1} \int_{0}^{x} \cos \left[(2 j+1)^{1 / 2}(x-t)\right] t^{2} h_{j}(t) d t .
$$

Then we have, using (61),

$$
\begin{aligned}
\int y^{2}\left(h_{j}^{\prime}(y)\right)^{2} f_{Y}(y) d y \geq & c^{\prime} \int_{a^{\prime}}^{b^{\prime}}\left(h_{j}^{\prime}\right)^{2}(y) d y \\
\geq & c^{\prime}(2 j+1) \lambda_{j}^{2} \int_{a^{\prime}}^{b^{\prime}} \sin ^{2}\left((2 j+1)^{1 / 2} y-\frac{j \pi}{2}\right) d y \\
& \quad-2 c^{\prime} \lambda_{j} \int_{a^{\prime}}^{b^{\prime}} \sin \left((2 j+1)^{1 / 2} y-\frac{j \pi}{2}\right) \xi_{j}^{\prime}(y) d y .
\end{aligned}
$$

We have $j^{-3 / 4} c_{1} / \sqrt{\pi} \leq \frac{2 \lambda_{j}}{(2 j+1)^{1 / 2}} \leq j^{-3 / 4} \sqrt{2 / \pi} c_{2}$ and

$$
\left|\lambda_{j} \int_{a}^{b} \sin \left((2 j+1)^{1 / 2} x-\frac{j \pi}{2}\right) \xi_{j}^{\prime}(x) d x\right| \leq \sqrt{2 j+1} \lambda_{j} \int_{a}^{b} \frac{|x|^{5 / 2}}{\sqrt{10}} d x \lesssim j^{1 / 4}
$$

Thus, the second term is lower bounded by $-O\left(j^{1 / 4}\right)$. For the first term, $\lambda_{j}^{2} \geq j^{-1 / 2} c_{1}^{2} / \pi$ and

$$
(2 j+1) \lambda_{j}^{2} \int_{a}^{b} \sin ^{2}\left((2 j+1)^{1 / 2} x-\frac{j \pi}{2}\right) d x=(2 j+1) \lambda_{j}^{2}\left\{\frac{b-a}{2}+O\left(\frac{1}{\sqrt{j}}\right)\right\} .
$$


Therefore, as $\lambda_{j}^{2}(2 j+1) \geq C \sqrt{j}$,

$$
\int y^{2}\left(h_{j}^{\prime}(y)\right)^{2} f(y) d y \geq c\left[\sqrt{j}\left(1+O\left(j^{-1 / 2}\right)\right)-j^{1 / 4}\right]
$$

Consequently, for $j$ large enough, $\int y^{2}\left(h_{j}^{\prime}(y)\right)^{2} f_{Y}(y) d y \geq c^{\prime} j^{1 / 2}$. This implies, as $V_{m}^{X} \lesssim m^{5 / 6}$ $(5 / 6<3 / 2), V_{m}=\sum_{j=0}^{m-1} \mathbb{E}\left[\left(Y_{1} h_{j}^{\prime}\left(Y_{1}\right)\right)^{2}\right]+V_{m}^{X} \geq c m^{3 / 2}$.

7.12. Proof of Theorem 4.1. Recall that $S_{m}$ is the space spanned by $\left\{\psi_{0}, \ldots, \psi_{m-1}\right\}$ and $B_{m}=\left\{t \in S_{m},\|t\|=1\right\}$. We have $\hat{f}_{m}=\arg \min _{t \in S_{m}} \gamma_{n}(t)$ where

$$
\gamma_{n}(t)=\|t\|^{2}-2 n^{-1} \sum_{i=1}^{n} \phi_{t}\left(Y_{i}\right), \quad \phi_{t}\left(Y_{i}\right):=Y_{i} t^{\prime}\left(Y_{i}\right)+t\left(Y_{i}\right)
$$

and $\gamma_{n}\left(\hat{f}_{m}\right)=-\left\|\hat{f}_{m}\right\|^{2}$. Now, we write, for two functions $t, s \in \mathbb{L}^{2}(\mathbb{R})$,

$$
\gamma_{n}(t)-\gamma_{n}(s)=\|t-f\|^{2}-\|s-f\|^{2}-2 \nu_{n}(t-s)
$$

where

$$
\nu_{n}(t)=\frac{1}{n} \sum_{i=1}^{n}\left[\phi_{t}\left(Y_{i}\right)-\langle t, f\rangle\right]
$$

recall that $\langle t, f\rangle=\mathbb{E}\left(\phi_{t}\left(Y_{1}\right)\right)$. Then, for any $m \in \mathcal{M}_{n}=\left\{1 \leq m \leq m_{n}\right\}$, and any $f_{m} \in S_{m}$,

$$
\gamma_{n}\left(\hat{f}_{\hat{m}}\right)+\widehat{\operatorname{pen}}(\hat{m}) \leq \gamma_{n}\left(f_{m}\right)+\widehat{\operatorname{pen}}(m) .
$$

This yields

$$
\left\|\hat{f}_{\hat{m}}-f\right\|^{2} \leq\left\|f-f_{m}\right\|^{2}+\widehat{\operatorname{pen}}(m)-\widehat{\operatorname{pen}}(\hat{m})+2 \nu_{n}\left(\hat{f}_{\hat{m}}-f_{m}\right)
$$

We use that

$$
2 \nu_{n}\left(\hat{f}_{\hat{m}}-f_{m}\right) \leq 4 \sup _{t \in B_{m \vee \hat{m}}}\left[\nu_{n}(t)\right]^{2}+\frac{1}{4}\left\|\hat{f}_{\hat{m}}-f_{m}\right\|^{2}
$$

and some classical algebra to obtain:

$$
\begin{aligned}
\frac{1}{2}\left\|\hat{f}_{\hat{m}}-f\right\|^{2} \leq & \frac{3}{2}\left\|f-f_{m}\right\|^{2}+\widehat{\operatorname{pen}}(m)+4\left(\sup _{t \in B_{m \vee \hat{m}}}\left[\nu_{n}(t)\right]^{2}-p(m \vee \hat{m})\right){ }_{+} \\
& +(4 p(m \vee \hat{m})-\operatorname{pen}(\hat{m}))+(\operatorname{pen}(\hat{m})-\widehat{\operatorname{pen}}(\hat{m})) .
\end{aligned}
$$

Lemma 7.6. Assume that $\mathbb{E}\left(X_{1}+\left(1 / \sqrt{X_{1}}\right)\right)<+\infty$ in the Laguerre case, $\mathbb{E}\left(\left|X_{1}\right|\right)<+\infty$ in the Hermite case, and that $m_{n} \leq n^{\beta}$ where $\beta=1 / 3$ for Laguerre and $\beta=6 / 17$ for Hermite. Then for $p(m)=4 V_{m} / n$, we have

$$
\begin{gathered}
\mathbb{E}\left(\sup _{t \in B_{m \vee \hat{m}}}\left[\nu_{n}(t)\right]^{2}-p(m \vee \hat{m})\right)_{+} \leq \frac{c}{n}, \\
\text { and } \quad \mathbb{E}(\operatorname{pen}(\hat{m})-\widehat{\operatorname{pen}}(\hat{m}))_{+} \leq \frac{1}{2} \mathbb{E}(\operatorname{pen}(\hat{m}))+\frac{c^{\prime} \log (n)}{n} .
\end{gathered}
$$

where $c$ and $c^{\prime}$ are positive constants. 
Taking expectation in (64) and using Lemma 7.6 yields

$$
\begin{aligned}
\frac{1}{2} \mathbb{E}\left(\left\|\hat{f}_{\hat{m}}-f\right\|^{2}\right) \leq \frac{3}{2} \| f- & f_{m} \|^{2}+\operatorname{pen}(m)+\mathbb{E}(4 p(m \vee \hat{m})-\operatorname{pen}(\hat{m}))+\frac{1}{2} \mathbb{E}(\operatorname{pen}(\hat{m})) \\
& +c^{\prime} \frac{\log (n)}{n}+\frac{4 c}{n} .
\end{aligned}
$$

Now we note that, for $\kappa \geq 32:=\kappa_{0}, 4 p(m \vee \hat{m})-\frac{1}{2} \operatorname{pen}(\hat{m}) \leq \frac{1}{2} \operatorname{pen}(m)$. Finally, we get, for all $m \in \mathcal{M}_{n}, \mathbb{E}\left(\left\|\hat{f}_{\hat{m}}-f\right\|^{2}\right) \leq 3\left\|f-f_{m}\right\|^{2}+3 \operatorname{pen}(m)+c^{\prime \prime} \frac{\log (n)}{n}$, which ends the proof.

\subsection{Proof of Lemma 7.6.}

7.13.1. Proof of (65). Note that

$$
\mathbb{E}\left(\sup _{t \in B_{m \vee \hat{m}}}\left[\nu_{n}(t)\right]^{2}-p(m \vee \hat{m})\right) \leq \sum_{m^{\prime} \in \mathcal{M}_{n}} \mathbb{E}\left(\sup _{t \in B_{m \vee m^{\prime}}}\left[\nu_{n}(t)\right]^{2}-p\left(m \vee m^{\prime}\right)\right)_{+} .
$$

We apply the Talagrand Inequality (see Theorem B.1):

$$
\mathbb{E}\left(\sup _{t \in B_{m}}\left[\nu_{n}(t)\right]^{2}-4 H^{2}\right)_{+} \leq \frac{C_{1}}{n}\left(v^{2} e^{-C_{2} \frac{n H^{2}}{v^{2}}}+\frac{M_{1}^{2}}{n} e^{-C_{3} \frac{n H}{M_{1}}}\right)
$$

where $H, v, M_{1}$ are such that $\mathbb{E}\left(\sup _{t \in B_{m}}\left[\nu_{n}(t)\right]^{2}\right) \leq H^{2}, \sup _{t \in B_{m}} \operatorname{Var}\left(\phi_{t}\left(Y_{1}\right)\right) \leq v^{2}$ and $\sup _{t \in B_{m}} \sup _{y}\left|\phi_{t}(y)\right| \leq$ $M_{1}$. We have

$$
\mathbb{E}\left(\sup _{t \in B_{m}}\left[\nu_{n}(t)\right]^{2}\right) \leq \sum_{j=0}^{m-1} \mathbb{E}\left[\nu_{n}^{2}\left(\psi_{j}\right)\right]=\frac{V_{m}}{n}:=H^{2} .
$$

Using Lemma 4.1, we have

$$
\sup _{t \in B_{m}} \operatorname{Var}\left(\phi_{t}\left(Y_{1}\right)\right) \leq \sup _{t \in B_{m}} \mathbb{E}\left(\phi_{t}^{2}\left(Y_{1}\right)\right) \leq \sup _{t \in B_{m}}\left[\mathbb{E}\left(Y_{1}^{2}\left[t^{\prime}\left(Y_{1}\right)\right]^{2}\right)+\mathbb{E}\left(t^{2}\left(X_{1}\right)\right)\right] .
$$

For any $t \in B_{m}$,

$$
\mathbb{E}\left(t^{2}\left(X_{1}\right)\right) \leq\|t\|_{\infty} \int|t| f \leq\|t\|_{\infty}\|f\| \lesssim \sqrt{m}\|f\|
$$

We need a specific study for the term $\sup _{t \in B_{m}} \mathbb{E}\left(Y_{1}^{2}\left[t^{\prime}\left(Y_{1}\right)\right]^{2}\right)$.

Laguerre case. From formula (48), we get

$$
y t^{\prime}(y)=-\sum_{j=0}^{m-1} a_{j}(t) y \varphi_{j}(y)-\sum_{j=0}^{m-1} a_{j}(t) \sqrt{2 j y} \varphi_{j-1}^{(1)}(y)
$$

By (13) and (14), $0 \leq y f_{Y}(y) \leq 1$ and $0 \leq y^{2} f_{Y}(y)=\int \frac{y^{2}}{x} \mathbf{1}_{x \geq y} f(x) d x \leq \mathbb{E}\left(X_{1}\right)$. Thus, using the orthonormality of $\left(\varphi_{j}\right)_{0 \leq j \leq m-1}$ and $\left(\varphi_{j}^{(1)}\right)_{0 \leq j \leq m-1}$, we have, for $t \in B_{m}$,

$$
\begin{aligned}
\mathbb{E}\left[\left(Y_{1} t^{\prime}\left(Y_{1}\right)\right)^{2}\right] & \leq 2 \int_{0}^{+\infty}\left(\sum_{j=0}^{m-1} a_{j}(t) \varphi_{j}(y)\right)^{2} y^{2} f_{Y}(y) d y+2 \int_{0}^{+\infty}\left(\sum_{j=0}^{m-1} a_{j}(t) \sqrt{2 j} \varphi_{j-1}^{(1)}(y)\right)^{2} y f_{Y}(y) d y \\
& \leq 2 \mathbb{E}\left(X_{1}\right)+4 m .
\end{aligned}
$$

Thus $\sup _{t \in B_{m}} \operatorname{Var}\left(\phi_{t}\left(Y_{1}\right)\right) \leq C m:=v^{2}$ where $C$ depends on $\mathbb{E}\left(X_{1}\right)$ and $\|f\|$.

Hermite case. By Lemma 4.1,2), we have $\sup _{t \in B_{m}} \mathbb{E}\left[\left(Y_{1} t^{\prime}\left(Y_{1}\right)\right)^{2}\right] \leq \sup _{t \in B_{m}} \mathbb{E}\left(\left|X_{1}\right|\right)\left\|t^{\prime}\right\|^{2}$ and by (52) we easily get that $\left\|t^{\prime}\right\|^{2} \leq 2 m$. Thus $v^{2}=C m$ where $C$ depends on $\mathbb{E}\left(\left|X_{1}\right|\right)$ and $\|f\|$. 
Next we note that, using Lemma 7.1 in Laguerre case and Lemma 7.3-(2) in Hermite case, we get, recalling that $\phi_{t}(y)=y t^{\prime}(y)+t(y)$,

$$
\sup _{t \in B_{m}} \sup _{y}\left|\phi_{t}(y)\right| \leq c m^{\omega}:=M_{1}
$$

with $\omega=3 / 2$ in Laguerre case and $\omega=17 / 12$ in Hermite case. Therefore in both cases, by using condition (24), $M_{1}^{2} / n \leq 1$ and we obtain

$$
\mathbb{E}\left(\sup _{t \in B_{m}}\left[\nu_{n}(t)\right]^{2}-4 \frac{V_{m}}{n}\right)_{+} \leq \frac{c_{1}}{n}\left(m e^{-C_{2}^{\prime} \frac{V_{m}}{m}}+e^{-C_{3}^{\prime} \frac{\sqrt{n V m}}{m^{3 / 2}}}\right) .
$$

Therefore as $V_{m} \gtrsim m^{3 / 2}$ under our assumptions, we get, using that $m^{3 / 4-3 / 2}=m^{-3 / 4} \geq n^{-9 / 34}$ under $m_{n} \leq n^{6 / 17}$ (Hermite case, which contains the Laguerre case $m_{n} \leq n^{1 / 3}$ ),

$$
\mathbb{E}\left(\sup _{t \in B_{m}}\left[\nu_{n}(t)\right]^{2}-4 \frac{V_{m}}{n}\right)_{+} \leq \frac{c_{1}^{\prime}}{n}\left(m e^{-c_{2}^{\prime} \sqrt{m}}+e^{-c_{3}^{\prime} n^{4 / 17}}\right) .
$$

Choosing $p(m)=4 V_{m} / n$ and using Inequality (67) yields (65).

7.13.2. Proof of (66). We proceed in this proof similarly to Massart (2007), chapter 7 (see Theorem 7.7). Let us define

$$
Z_{i}^{(m)}:=\sum_{j=0}^{m-1} \phi_{\psi_{j}}^{2}\left(Y_{i}\right), \quad \widehat{V}_{m}=\frac{1}{n} \sum_{i=1}^{n} Z_{i}^{(m)}
$$

Bernstein's Inequality (see (2.23) in Massart (2007)) writes $\mathbb{P}\left(\left|S_{n} / n\right| \geq \sqrt{2 s^{2} x / n}+b x /(3 n)\right) \leq$ $2 e^{-x}$ for $S_{n}=\sum_{i=1}^{n}\left(U_{i}-\mathbb{E}\left(U_{i}\right)\right)$, and i.i.d. $U_{i}$ 's, with $\operatorname{Var}\left(U_{1}\right) \leq s^{2},\left|U_{i}\right| \leq b$. We consider $U_{i}=Z_{i}^{(m)}$ and $x=2 \log (n)$. We have, using Lemma 7.1 in Laguerre case and Lemma 7.3-(2) in Hermite case,

$$
\begin{aligned}
\operatorname{Var}\left(Z_{i}^{(m)}\right) \leq \mathbb{E}\left[\left(Z_{i}^{(m)}\right)^{2}\right] & \leq V_{m}\left\|\sum_{j=0}^{m-1} \phi_{\psi_{j}}^{2}\right\|_{\infty} \leq C m^{1 / \beta} V_{m}:=s^{2}, \\
\text { and }\left|Z_{i}^{(m)}\right| & \leq\left\|\sum_{j=0}^{m-1} \phi_{\psi_{j}}^{2}\right\|_{\infty} \leq m^{1 / \beta}:=b
\end{aligned}
$$

Let us define the set

$$
\Omega=\left\{\forall m \in \mathcal{M}_{n}, \quad \frac{1}{n}\left|\sum_{i=1}^{n}\left(Z_{i}^{(m)}-\mathbb{E}\left(Z_{i}^{(m)}\right)\right)\right| \leq \sqrt{2 V_{m} C m^{1 / \beta} \frac{2 \log (n)}{n}}+C m^{1 / \beta} \frac{2 \log (n)}{3 n}\right\} .
$$

Now, applying the Bernstein inequality gives $\mathbb{P}\left(\Omega^{c}\right) \leq \sum_{m \in \mathcal{M}_{n}} 2 e^{-2 \log (n)} \leq c / n$. We write

$$
\begin{aligned}
& \mathbb{E}(\operatorname{pen}(\hat{m})-\widehat{\operatorname{pen}}(\hat{m}))_{+} \leq \mathbb{E}\left[(\operatorname{pen}(\hat{m})-\widehat{\operatorname{pen}}(\hat{m}))_{+} \mathbf{1}_{\Omega}\right]+\mathbb{E}\left[(\operatorname{pen}(\hat{m})-\widehat{\operatorname{pen}}(\hat{m}))_{+} \mathbf{1}_{\Omega^{c}}\right] \\
& \operatorname{On} \Omega, \quad\left|\widehat{V}_{\hat{m}}-V_{\hat{m}}\right| \leq \sqrt{2 V_{m} C m^{1 / \beta} \frac{2 \log (n)}{n}}+C m^{1 / \beta} \frac{2 \log (n)}{3 n} \\
& \leq \frac{1}{2} V_{\hat{m}}+\frac{8}{3} C \frac{\hat{m}^{1 / \beta} \log (n)}{n}
\end{aligned}
$$


using that $2 x y \leq x^{2}+y^{2}$ applied to $\sqrt{2 V A}=2 \sqrt{V / 2} \sqrt{A} \leq V / 2+A$ with $V=V_{\hat{m}}$ and $A=2 C m^{1 / \beta} \log (n) / n$. Thus, as by definition of $\mathcal{M}_{n}, \hat{m}^{1 / \beta} \leq m_{n}^{1 / \beta} \leq n$,

$$
\mathbb{E}\left[(\operatorname{pen}(\hat{m})-\widehat{\operatorname{pen}}(\hat{m}))_{+} \mathbf{1}_{\Omega}\right]+\leq \frac{1}{2} \mathbb{E}(\operatorname{pen}(\hat{m}))+c \frac{\log (n)}{n} .
$$

On the other hand, on $\Omega^{c}, \mathbb{E}\left[(\operatorname{pen}(\hat{m})-\widehat{\operatorname{pen}}(\hat{m}))_{+} \mathbf{1}_{\Omega^{c}}\right] \leq 2 \kappa \mathbb{P}\left(\Omega^{c}\right) \leq c / n$. Thus ( 66) follows from the last two inequalities.

7.14. Proof of Proposition 4.4. We have for all $\ell>0$,

$$
\mathbb{E}\left[\left\|\tilde{f}_{\ell}-f\right\|^{2}\right]=\left\|f-\bar{f}_{\ell}\right\|^{2}+\mathbb{E}\left[\left\|\tilde{f}_{\ell}-\bar{f}_{\ell}\right\|^{2}\right] .
$$

Next,

$$
\begin{aligned}
\mathbb{E}\left[\left\|\tilde{f}_{\ell}-\bar{f}_{\ell}\right\|^{2}\right] & =\frac{1}{2 \pi} \mathbb{E}\left[\left\|\tilde{f}_{\ell}^{*}-\bar{f}_{\ell}^{*}\right\|^{2}\right]=\frac{1}{2 \pi n} \int_{-\pi \ell}^{\pi \ell} \operatorname{Var}\left(e^{i u Y_{1}}+i u Y_{1} e^{i u Y_{1}}\right) d u \\
& =\frac{1}{2 \pi n} \int_{-\pi \ell}^{\pi \ell} \mathbb{E}\left|e^{i u Y_{1}}+i u Y_{1} e^{i u Y_{1}}\right|^{2} d u-\frac{\left\|\bar{f}_{\ell}\right\|^{2}}{n} \\
& =\frac{1}{2 \pi n} \int_{-\pi \ell}^{\pi \ell}\left(1+u^{2} \mathbb{E}\left(Y_{1}^{2}\right)\right) d u-\frac{\left\|\bar{f}_{\ell}\right\|^{2}}{n} \\
& =\frac{\ell}{n}\left(1+\frac{\pi^{2}}{3} \mathbb{E}\left(Y_{1}^{2}\right) \ell^{2}\right)-\frac{\left\|\bar{f}_{\ell}\right\|^{2}}{n} .
\end{aligned}
$$

Gathering the two terms gives Inequality (30). On the other hand, we have for all $\ell>0$,

$$
\mathbb{E}\left[\left\|\tilde{f}_{\ell}^{(n)}-f\right\|^{2}\right] \leq\left\|f-\bar{f}_{\ell}\right\|^{2}+2\left\|\bar{f}_{\ell}-\mathbb{E} \tilde{f}_{\ell}^{(n)}\right\|^{2}+2 \mathbb{E}\left[\| \tilde{f}_{\ell}^{(n)}-\mathbb{E}\left(\tilde{f}_{\ell}^{(n)} \|^{2}\right]:=T_{1}+T_{2}+T_{3} .\right.
$$

The term $T_{1}$ is the same bias term as before. The term $T_{2}$ is bounded in Belomestny et al. (2017), Proposition 3.1 and we have the bound $T_{2} \leq 4 \ell^{2}\left(M_{2}+1\right) / L_{n}$. This term is $O(\ell / n)$ if $\ell \leq n$ and $L_{n} \geq n^{2}$.

For $T_{3}$, using Lemma 4.1 with $t=\theta_{\ell, j}^{2}$, we write that

$$
\begin{aligned}
T_{3} & =\sum_{|j| \leq L_{n}} \operatorname{Var}\left(\tilde{\tilde{a}}_{\ell, j}\right)=\frac{1}{n} \sum_{|j| \leq L_{n}} \operatorname{Var}\left[\theta_{\ell, j}\left(Y_{1}\right)+Y_{1} \theta_{\ell, j}^{\prime}\left(Y_{1}\right)\right] \\
& \leq \frac{1}{n} \sum_{|j| \leq L_{n}} \mathbb{E}\left[\theta_{\ell, j}^{2}\left(Y_{1}\right)+2 Y_{1} \theta_{\ell, j}\left(Y_{1}\right) \theta_{\ell, j}^{\prime}\left(Y_{1}\right)+Y_{1}^{2}\left(\theta_{\ell, j}^{\prime}\left(Y_{1}\right)\right)^{2}\right] \\
& =\frac{1}{n} \sum_{|j| \leq L_{n}}\left[\mathbb{E}\left[\theta_{\ell, j}^{2}\left(X_{1}\right)\right]+\mathbb{E}\left[Y_{1}^{2}\left(\theta_{\ell, j}^{\prime}\left(Y_{1}\right)\right)^{2}\right]\right]
\end{aligned}
$$

Next, we know that $\sum_{|j| \leq L_{n}} \theta_{\ell, j}^{2} \leq \sum_{j \in \mathbb{Z}} \theta_{\ell, j}^{2}=\ell$. As moreover

$$
\sum_{j \in \mathbb{Z}}\left[\theta_{\ell, j}^{\prime}(x)\right]^{2}=\frac{1}{2 \pi} \sum_{j \in \mathbb{Z}}\left[\left\langle\frac{\theta_{\ell, j}^{*}}{\sqrt{2 \pi}}, t e^{-i t x}\right\rangle\right]^{2}=\frac{1}{2 \pi} \int_{-\pi \ell}^{\pi \ell} t^{2} d t=\frac{\pi^{2} \ell^{3}}{3},
$$

we obtain

$$
T_{3} \leq \frac{\ell}{n}\left(1+\mathbb{E}\left(Y_{1}^{2}\right) \frac{\pi^{2} \ell^{2}}{3}\right) .
$$

Plugging this bound in Inequality (68) gives Inequality (31). 
7.15. Proof of Proposition 5.1. The risk of the estimator can be written as usual

$$
\left\|\tilde{f}_{m}-f\right\|^{2}=\left\|f-f_{m}\right\|^{2}+\left\|\tilde{f}_{m}-f_{m}\right\|^{2}
$$

where $f_{m}=\sum_{j=0}^{m-1} a_{j}(f) \varphi_{j}$ is the projection of $f$ on $S_{m}=\operatorname{span}\left(\varphi_{0}, \ldots, \varphi_{m-1}\right)$ and $\left\|f-f_{m}\right\|^{2}$ is the square bias term. Next we have

$$
\left\|\tilde{f}_{m}-f_{m}\right\|^{2}=\sum_{j=0}^{m-1}\left(\tilde{a}_{j}-a_{j}(f)\right)^{2}=\left\|\boldsymbol{\Sigma}_{m}^{-1}\left(\overrightarrow{\hat{a}}(Z)_{m-1}-\mathbb{E}\left(\overrightarrow{\hat{a}}(Z)_{m-1}\right)\right)\right\|_{2}^{2},
$$

where $\|\vec{x}\|_{2}$ denotes the Euclidean norm of the $m$-vector $\vec{x}$. So,

$$
\begin{aligned}
\mathbb{E}\left(\left\|\tilde{f}_{m}-f_{m}\right\|^{2}\right) & \leq\left\|\boldsymbol{\Sigma}_{m}^{-1}\right\|_{\mathrm{op}}^{2} \mathbb{E}\left(\left\|\overrightarrow{\hat{a}}(Z)_{m-1}-\mathbb{E}\left(\overrightarrow{\hat{a}}(Z)_{m-1}\right)\right\|_{2}^{2}\right) \\
& \leq\left\|\boldsymbol{\Sigma}_{m}^{-1}\right\|_{\mathrm{op}}^{2} \sum_{j=0}^{m-1} \operatorname{Var}\left(\hat{a}_{j}(Z)\right)=\frac{1}{n}\left\|\boldsymbol{\Sigma}_{m}^{-1}\right\|_{\mathrm{op}}^{2} \sum_{j=0}^{m-1} \operatorname{Var}\left(\varphi_{j}\left(Z_{1}\right)\right) \\
& \leq \frac{1}{n}\left\|\boldsymbol{\Sigma}_{m}^{-1}\right\|_{\mathrm{op}}^{2} \sum_{j=0}^{m-1} \mathbb{E}\left(\varphi_{j}^{2}\left(Z_{1}\right)\right) \leq \frac{c \sqrt{m}\left\|\boldsymbol{\Sigma}_{m}^{-1}\right\|_{\mathrm{op}}^{2}}{n}
\end{aligned}
$$

using Lemma 7.2. On the other hand,

$$
\begin{aligned}
\mathbb{E}\left(\left\|\tilde{f}_{m}-f_{m}\right\|^{2}\right) & =\frac{1}{n} \sum_{\ell} \operatorname{Var}\left(\sum_{j}\left[\boldsymbol{\Sigma}_{m}^{-1}\right]_{\ell, j} \varphi_{j}\left(Z_{1}\right)\right) \leq \frac{1}{n} \sum_{\ell} \mathbb{E}\left[\left(\sum_{j}\left[\boldsymbol{\Sigma}_{m}^{-1}\right]_{\ell, j} \varphi_{j}\left(Z_{1}\right)\right)^{2}\right] \\
\leq & \frac{\left\|f_{Z}\right\|_{\infty}}{n} \sum_{\ell} \int\left(\sum_{j}\left[\boldsymbol{\Sigma}_{m}^{-1}\right]_{\ell, j} \varphi_{j}(z)\right)^{2} d z \\
& =\frac{\left\|f_{Z}\right\|_{\infty}}{n} \sum_{\ell} \sum_{j}\left[\boldsymbol{\Sigma}_{m}^{-1}\right]_{\ell, j}^{2} \leq \frac{\left\|f_{\Sigma}\right\|_{\infty}}{n}\left\|\boldsymbol{\Sigma}_{m}^{-1}\right\|_{F}^{2} .
\end{aligned}
$$

Combining the previous bounds implies the result.

7.16. Proof of Proposition 5.2. Let us denote by $\check{f}_{\ell, m}(x)=\sum_{j=0}^{m-1} \check{a}_{j, \ell} h_{j}(x)$ and $\mathbb{E}\left(\check{f}_{\ell, m}\right)=$ $f_{\ell, m}$ where $f_{\ell, m}=\sum_{j=0}^{m-1} a_{j, \ell} h_{j}$,

$$
a_{j, \ell}(f)=\mathbb{E}\left(\check{a}_{j, \ell}\right)=\frac{(-i)^{j}}{\sqrt{2 \pi}} \int_{-\pi \ell}^{\pi \ell} f^{*}(t) h_{j}(t) d t .
$$

We have

$$
\begin{aligned}
\mathbb{E}\left(\left\|\check{f}_{\ell, m}-f\right\|^{2}\right) & =\left\|f-f_{\ell, m}\right\|^{2}+\mathbb{E}\left(\left\|\check{f}_{\ell, m}-f_{\ell, m}\right\|^{2}\right) \\
& =\left\|f-f_{m}\right\|^{2}+\sum_{j=0}^{m-1}\left(a_{j}(f)-a_{j, \ell}(f)\right)^{2}+\sum_{j=0}^{m-1} \operatorname{Var}\left(\check{a}_{j, \ell}\right) \\
& =\sum_{j \geq m} a_{j}^{2}(f)+\sum_{j=0}^{m-1}\left(a_{j}(f)-a_{j, \ell}(f)\right)^{2}+\frac{1}{n} \sum_{j=0}^{m-1} \operatorname{Var}\left(\frac{(-i)^{j}}{\sqrt{2 \pi}} \int_{-\pi \ell}^{\pi \ell} e^{i t Z_{1}} \frac{h_{j}(t)}{f_{\Sigma}^{*}(t)} d t\right)
\end{aligned}
$$

Then

$$
\sum_{j=0}^{m-1}\left(a_{j}(f)-a_{j, \ell}(f)\right)^{2}=\sum_{j=0}^{m-1} \frac{1}{2 \pi}\left|\int f^{*}(t) \mathbf{1}_{|t| \geq \pi \ell} h_{j}(t) d t\right|^{2} \leq \frac{1}{2 \pi} \int_{|t| \geq \pi \ell}\left|f^{*}(t)\right|^{2} d t .
$$


On the other hand, for the variance term, we have

$$
\begin{aligned}
\frac{1}{n} \sum_{j=0}^{m-1} \operatorname{Var}\left(\frac{(-i)^{j}}{\sqrt{2 \pi}} \int_{-\pi \ell}^{\pi \ell} e^{i t Z_{1}} \frac{h_{j}(t)}{f_{\Sigma}^{*}(t)} d t\right) & \leq \frac{1}{n} \sum_{j=0}^{m-1} \mathbb{E}\left(\left|\frac{(-i)^{j}}{\sqrt{2 \pi}} \int_{-\pi \ell}^{\pi \ell} e^{i t Z_{1}} \frac{h_{j}(t)}{f_{\Sigma}^{*}(t)} d t\right|^{2}\right) \\
& =\frac{1}{2 \pi n} \mathbb{E}\left(\sum_{j=0}^{m-1}\left|\int \frac{\mathbf{1}_{|t| \leq \pi \ell} e^{i t Z_{1}}}{f_{\Sigma}^{*}(t)} h_{j}(t) d t\right|^{2}\right) \\
& \leq \frac{1}{2 \pi n} \int \frac{\mathbf{1}_{|t| \leq \pi \ell}}{\left|f_{\Sigma}^{*}(t)\right|^{2}} d t .
\end{aligned}
$$

Therefore we obtain

$$
\mathbb{E}\left(\left\|\check{f}_{\ell, m}-f\right\|^{2}\right) \leq \sum_{j \geq m} a_{j}^{2}(f)+\frac{1}{2 \pi} \int_{|t| \geq \pi \ell}\left|f^{*}(t)\right|^{2} d t+\frac{1}{2 \pi n} \int \frac{\mathbf{1}_{|t| \leq \pi \ell}}{\left|f_{\Sigma}^{*}(t)\right|^{2}} d t .
$$

Choosing $\ell=\sqrt{m}$ gives the announced result.

7.17. Proof of Theorem 5.1. Let $\mathfrak{M}=\max \mathcal{M}_{n}$ the maximal element of the collection. We follow the lines of the proof of Theorem 4.1, with (62) replaced by

$$
\tilde{\gamma}_{n}(t)=\|t\|^{2}-2\left\langle t, \tilde{f}_{\mathfrak{M}}\right\rangle
$$

and $(63)$ by $\tilde{\nu}_{n}(t)=\left\langle t, \tilde{f}_{\mathfrak{M}}-f_{\mathfrak{M}}\right\rangle$. Note that for $t \in S_{m}$, then $\tilde{\nu}_{n}(t)=\left\langle t, \tilde{f}_{m}-f_{m}\right\rangle$. Then we get

$$
\left\|\tilde{f}_{\tilde{m}}-f\right\|^{2} \leq 3\left\|f-f_{m}\right\|^{2}+2 \widetilde{\operatorname{pen}}(m)+8\left(\sup _{t \in \mathcal{B}_{m, \tilde{m}}} \tilde{\nu}_{n}^{2}(t)-\tilde{p}(m, \tilde{m})\right)+8 \tilde{p}(m, \tilde{m})-2 \widetilde{\operatorname{pen}}(\tilde{m})
$$

with $\tilde{p}\left(m, m^{\prime}\right)$ satisfying $4 \tilde{p}\left(m, m^{\prime}\right) \leq \widetilde{\operatorname{pen}}(m)+\widetilde{\operatorname{pen}}\left(m^{\prime}\right)$ for $\kappa \geq \kappa_{0}$, where $\tilde{p}\left(m, m^{\prime}\right)$ and $\kappa_{0}$ are specified by the next Lemma.

Lemma 7.7. Under the assumptions of Theorem 5.1, for $m^{*}=m \vee m^{\prime}$ and

$$
\tilde{p}\left(m, m^{\prime}\right)=2\left(2 \vee\left\|f_{\Sigma}\right\|_{\infty}\right)\left(1+2 c \log \left(2+\left\|\Sigma_{m^{*}}^{1}\right\|_{F}^{2}\right)\right) \frac{\left\|\Sigma_{m^{*}}^{-1}\right\|_{F}^{2}}{n}, \quad c \geq \max \left(3 / b, 21^{2} / 2 b^{2}\right)
$$

where $b$ is a constant given in Theorem B.1, we have

$$
\mathbb{E}\left[\left(\sup _{\vec{t} \in B(\tilde{m}, m)} \tilde{\nu}_{n}^{2}(\vec{t})-\tilde{p}(m, \tilde{m})\right)_{+}\right] \leq \frac{K}{n}
$$

We obtain that $\forall m \in \mathcal{M}_{n}$,

$$
\mathbb{E}\left(\left\|\tilde{f}_{\tilde{m}}-f\right\|^{2}\right) \leq 3\left\|f-f_{m}\right\|^{2}+4 \widetilde{\operatorname{pen}}(m)+8 \frac{K}{n},
$$

which ends the proof of Theorem 5.1.

7.18. Proof of Lemma 7.7. The proof of (69) follows the line of the proof of Proposition 7.1 in Mabon (2015). We start as in the proof of Lemma 7.6 and compute the terms $H^{2}, v$ and $M$ of Theorem B.1. For $t \in B(\tilde{m}, m)$ and $m^{*}=m \vee m^{\prime}$, we get

$$
\mathbb{E}\left(\sup _{t \in B\left(m^{\prime}, m\right.} \tilde{\nu}_{n}^{2}(t)\right) \leq \sum_{j=0}^{m^{*}-1} \mathbb{E}\left(\tilde{\nu}_{n}^{2}\left(\varphi_{j}\right)\right) \leq \sum_{j=0}^{m^{*}-1} \mathbb{E}\left(\left\langle\varphi_{j}, \tilde{f}_{m^{*}}-f_{m^{*}}\right\rangle^{2}\right)=\mathbb{E}\left(\left\|\tilde{f}_{m^{*}}-f_{m^{*}}\right\|^{2}\right) .
$$


From Proposition 5.1, we deduce $H^{2}=\left(2 \vee\left\|f_{\Sigma}\right\|_{\infty}\right)\left\|\Sigma_{m^{*}}^{-1}\right\|_{F}^{2} / n$. Clearly, $v=n H^{2}$. Moreover

$$
\tilde{\nu}_{n}(t)=\frac{1}{n} \sum_{i=1}^{n}\left[\psi_{t}\left(Z_{i}\right)-\mathbb{E}\left(\psi_{t}\left(Z_{i}\right)\right)\right], \quad \psi_{t}(x)=\sum_{j=0}^{m^{*}-1}\left\langle t, \varphi_{j}\right\rangle\left[V_{m^{*}}^{-1} \vec{\varphi}_{m^{*}-1}(x)\right]_{j}
$$

where $\vec{\varphi}_{m-1}(x)={ }^{t}\left(\varphi_{0}(x), \ldots, \varphi_{m-1}(x)\right)$ and $[\vec{x}]_{j}$ denotes the $j$ th coordinate of vector $\vec{x}$. Thus

$$
\sup _{t \in B\left(m^{\prime}, m\right)} \sup _{x}\left|\psi_{t}(x)\right| \leq \sup _{x}\left\|\boldsymbol{\Sigma}_{m^{*}}^{-1} \vec{\varphi}_{m^{*}-1}(x)\right\|_{2} \leq\left\|\boldsymbol{\Sigma}_{m^{*}}^{-1}\right\|_{\text {op }} \sqrt{2 m^{*}}:=M .
$$

Let $\alpha\left(m^{*}\right)=c \log \left(2+\left\|\boldsymbol{\Sigma}_{m^{*}}^{1}\right\|_{F}^{2}\right)$, and let us apply Theorem B.1

$$
\begin{aligned}
& \mathbb{E}\left(\sup _{t \in B\left(m^{\prime}, m\right)} \tilde{\nu}_{n}^{2}(t)-2\left(1+2 \alpha\left(m^{*}\right)\right) H^{2}\right)_{+} \\
\leq & \frac{C}{n}\left(\left\|\boldsymbol{\Sigma}_{m^{*}}^{-1}\right\|_{F}^{2} \exp \left(-b \alpha\left(m^{*}\right)\right)+\frac{m^{*}\left\|\boldsymbol{\Sigma}_{m^{*}}^{-1}\right\|_{\mathrm{op}}^{2}}{n} \exp \left(-\frac{\sqrt{2} b}{7} \frac{\sqrt{\alpha\left(m^{*}\right) n}\left\|\boldsymbol{\Sigma}_{m^{*}}^{-1}\right\|_{F}}{\sqrt{m^{*}}\left\|\boldsymbol{\Sigma}_{m^{*}}^{-1}\right\|_{\mathrm{op}}}\right)\right) \\
\leq & \frac{C}{n}\left(\frac{1}{\left\|\boldsymbol{\Sigma}_{m^{*}}^{-1}\right\|_{F}^{2 b c-2}}+\left\|\boldsymbol{\Sigma}_{m^{*}}^{-1}\right\|_{F}^{2} \exp \left(-\frac{\sqrt{2} b}{7} \sqrt{\alpha\left(m^{*}\right) \log (n+2)}\right)\right),
\end{aligned}
$$

where we have used that $m^{*} \leq n / \log (n+2)$ and $\left\|\boldsymbol{\Sigma}_{m}^{-1}\right\|_{\text {op }}^{2} \leq\left\|\boldsymbol{\Sigma}_{m}^{-1}\right\|^{2}$. Therefore

$$
\mathbb{E}\left(\sup _{t \in B\left(m^{\prime}, m\right)} \tilde{\nu}_{n}^{2}(t)-2\left(1+2 \alpha\left(m^{*}\right)\right) H^{2}\right)_{+} \leq \frac{C}{n}\left(\frac{1}{\left\|\boldsymbol{\Sigma}_{m^{*}}^{-1}\right\|_{F}^{2 b c-2}}+\frac{1}{\left\|\boldsymbol{\Sigma}_{m^{*}}^{-1}\right\|_{F}^{\sqrt{2 c} b / 7-2}}\right) .
$$

For $c \geq \max \left(3 / b, 21^{2} / 2 b^{2}\right)$ and as $\left\|\boldsymbol{\Sigma}_{m}^{-1}\right\|_{F}^{2} \geq 2 m^{*} / a_{0}\left(f_{\Sigma}\right)$, we get

$$
\mathbb{E}\left(\sup _{t \in B\left(m^{\prime}, m\right)} \tilde{\nu}_{n}^{2}(t)-2\left(1+2 \alpha\left(m^{*}\right)\right) H^{2}\right)_{+} \leq \frac{C^{\prime}}{n} \frac{1}{\left(m^{*}\right)^{4}}
$$

so that

$$
\sum_{m^{\prime} \in \mathcal{M}_{n}} \mathbb{E}\left(\sup _{t \in B\left(m^{\prime}, m\right)} \tilde{\nu}_{n}^{2}(t)-2\left(1+2 \alpha\left(m^{*}\right)\right) H^{2}\right)_{+} \leq C^{\prime \prime} / n
$$

This concludes of Lemma 7.7.

\section{REFERENCES}

[1] Abbaszadeh, M., Chesneau, C. and Doosti, H. (2012) Nonparametric estimation of density under bias and multiplicative censoring via wavelet methods. Statist. Probab. Lett. 82, 932-941.

[2] Abbaszadeh, M., Chesneau, C. and Doosti, H. (2013) Multiplicative censoring: estimation of a density and its derivatives under the Lp-risk. REVSTAT 11, 255-276.

[3] Abramowitz, M. and Stegun, I. A. (1964) Handbook of mathematical functions with formulas, graphs, and mathematical tables. National Bureau of Standards Applied Mathematics Series, 55 For sale by the Superintendent of Documents, U.S. Government Printing Office, Washington, D.C.

[4] Askey, R. and Wainger, S. (1965) Mean convergence of expansions in Laguerre and Hermite series. Amer. J. Math. 87, 695-708.

[5] Asgharian, M., Carone, M., Fakoor, V. (2012) Large-sample study of the kernel density estimators under multiplicative censoring. Ann. Statist. 40, 159-187.

[6] Andersen, K. E. and Hansen, M. B. (2001) Multiplicative censoring: density estimation by a series expansion approach. J. Statist. Plann. Inference 98, 137-155.

[7] D. Belomestny, F. Comte and V. Genon-Catalot. (2016) Nonparametric Laguerre estimation in the multiplicative censoring model. Preprint.

[8] D. Belomestny, F. Comte and V. Genon-Catalot. (2017) Sobolev-Hermite versus Sobolev nonparametric density estimation on $\mathrm{R}$, to appear in The Annals of the Institute of Mathematical Statistics. 
[9] Bongioanni, B. and Torrea, J.L. (2006). Sobolev spaces associated to the harmonic oscillator. Proc.Indian Acad. Sci. (math. Sci.) 116 (3), 337-360.

[10] Bongioanni, B. and Torrea, J.L. (2009). What is a Sobolev space for the Laguerre function system? Studia Mathematica 192 (2), 147-172.

[11] Brunel, E., Comte, F. and Genon-Catalot, V. (2016) Nonparametric density and survival function estimation in the multiplicative censoring model. TEST 25, 570-590.

[12] Chesneau, C. (2013) Wavelet estimation of a density in a GARCH-type model. Comm. Statist. Theory Methods 42, 98-117.

[13] Comte, F., Cuenod, C.-A., Pensky, M. and Rozenholc, Y. (2017). Laplace deconvolution and its application to Dynamic Contrast Enhanced imaging. J. R. Stat. Soc. Ser. B, 79, 69-94.

[14] Comte, F. and Genon-Catalot, V. (2015) Adaptive Laguerre density estimation for mixed Poisson models. Electron. J. Stat. 9, 1113-1149.

[15] Comte, F., Rozenholc, Y. and Taupin, M.-L. (2006) Penalized contrast estimator for adaptive density deconvolution. Canad. J. Statist. 34, 431-452.

[16] Efromovich, S. (1999). Nonparametric curve estimation. Methods, theory, and applications. Springer Series in Statistics. Springer-Verlag, New York.

[17] Efromovich, S. (2009). Lower bound for estimation of Sobolev densities of order less 1/2. J. Statist. Plann. Inference 139, 2261-2268.

[18] van Es, B., Spreij, P. and van Zanten, H. (2003). Nonparametric volatility density estimation. Bernoulli 9, 451-465.

[19] van Es, B., Jongbloed, G. and van Zuijlen, M. (1998) Isotonic inverse estimators for nonparametric deconvolution. Ann. Statist. 26, 2395-2406.

[20] Jirak, M., Meister, A. and Reiss, M. (2014) Adaptive function estimation in nonparametric regression with one-sided errors. Ann. Statist. 42, 1970-2002.

[21] Jongbloed, G. (1998) Exponential deconvolution: two asymptotically equivalent estimators. Statist. Neerlandica 52, 6-17.

[22] Juditsky, A. and Lambert-Lacroix, S. (2004) On minimax density estimation on R. Bernoulli 10, $187-220$.

[23] Klein, T. and Rio, E. (2005). Concentration around the mean for maxima of empirical processes. Ann. Probab. 33 1060-1077.

[24] Mabon, G. (2017). Adaptive deconvolution on the nonnegative real line. Scandinavian Journal of Statistics, 44, 707-740.

[25] Massart, P. (2007) Concentration inequalities and model selection. Lectures from the 33rd Summer School on Probability Theory held in Saint-Flour, July 6-23, 2003. With a foreword by Jean Picard. Lecture Notes in Mathematics, 1896. Springer, Berlin, 2007.

[26] Rigollet, P. (2006) Adaptive density estimation using the blockwise Stein method. Bernoulli 12, 351-370.

[27] Shen, J. (2000). Stable and efficient spectral methods in unbounded domains using Laguerre functions. SIAM J. Numer. Anal. 38, 1113-1133.

[28] Schipper, M. (1996). Optimal rates and constants in L2-minimax estimation of probability density functions. Mathematical Methods of Statistics, 5, 253-274.

[29] Vardi, Y. (1989) Multiplicative censoring, renewal processes, deconvolution and decreasing density: nonparametric estimation. Biometrika 76, 751-761.

[30] Vardi, Y. and Zhang, C.-H. (1992) Large sample study of empirical distributions in a random-multiplicative censoring model. Ann. Statist. 20, 1022-1039.

[31] Vareschi, T. (2015) Noisy Laplace deconvolution with error in the operator. J. Statist. Plann. Inference $\mathbf{1 5 7 / 1 5 8 , 1 6 - 3 5 . ~}$

[32] Zhang, Z. and Su, W. (2017) A new efficient method for estimating the Gerber-Shiu function in the classical risk model, to appear in Scandinavian Actuarial Journal, http://dx.doi.org/10.1080/03461238.2017.1371068

\section{Appendix A. Regularity properties of functions in Sobolev-Laguerre And Sobolev-Hermite SPACES}

For this appendix, we refer to Bongionanni and Torrea $(2006,2009)$, Belomestny et al. $(2016,2017)$ and Comte and Genon-Catalot (2015).

Laguerre case. For $a>0$, consider the functions

$$
\varphi_{k}^{(\delta, a)}(x)=a^{(\delta+1) / 2}\left(\frac{k !}{\Gamma(k+\delta+1)}\right)^{1 / 2} \exp (-a x / 2) x^{\delta / 2} L_{k}^{(\delta}(a x), \quad k \geq 0 .
$$


This sequence is an orthonormal basis of $\mathbb{L}^{2}\left(\mathbb{R}^{+}\right)$. We set $a_{k}^{(\delta, a)}(f)=\left\langle f, \varphi_{k}^{(\delta, a)}\right\rangle$ and define the Sobolev-Laguerre space with regularity index $s$ and scale parameter $a$ by:

$$
W_{\delta, a}^{s}=\left\{f:(0,+\infty) \rightarrow \mathbb{R}, f \in L^{2}((0,+\infty)), \sum_{k \geq 0} k^{s}\left(a_{k}^{(\delta, a)}(f)\right)^{2}<+\infty\right\} .
$$

For $f$ a derivable function on $(0,+\infty)$, consider the operator

$$
\partial_{a}^{\delta}(f)(x)=\sqrt{x} f^{\prime}(x)+\left(\frac{a}{2} \sqrt{x}-\frac{\delta}{2 \sqrt{x}}\right) f(x) .
$$

If $g=\exp (a x / 2) x^{-\delta / 2} f$ and $f$ is $m+1$ times derivable, then, the following holds:

$$
\partial_{a}^{\delta+m} \ldots \circ \partial_{a}^{\delta+1} \circ \partial_{a}^{\delta}(f)(x)=g^{(m+1)}(x) \exp (-a x / 2) x^{(\delta+m+1) / 2} .
$$

For $s$ integer, the space $W_{\delta, a}^{s}$ is exactly the space of functions $f:(0,+\infty) \rightarrow \mathbb{R}, f \in L^{2}((0,+\infty))$ such tht $f$ is $s-1$ derivable, $f^{(s-1)}$ is absolutely continuous and $\partial_{a}^{\delta+m} \ldots \circ \partial_{a}^{\delta+1} \circ \partial_{a}^{\delta}(f)$ belongs to $L^{2}((0,+\infty))$ for $m=0,1, \ldots, s-1$.

The choice $a=2, \delta=0$ seems the simplest one, and $W_{L}^{s}=W_{0,2}^{s}$.

Hermite case. For $s$ integer, $f \in W_{H}^{s}$ holds if and only if $f$ admits derivatives up to order $s$ which satisfy $f, f^{\prime}, \ldots, f^{(s)}, x^{s-\ell} f^{(\ell)}, \ell=0, \ldots, s-1$ belong to $\mathbb{L}^{2}(\mathbb{R})$. The usual Sobolev space with regularity index $s$ is defined by

$$
\mathcal{W}^{s}=\left\{f \in \mathbb{L}^{2}(\mathbb{R}),\|f\|_{s, s o b}^{2}=\int_{\mathbb{R}}\left(1+t^{2 s}\right)\left|f^{*}(t)\right|^{2} d t<+\infty\right\}
$$

If $s$ is integer, then

$$
\begin{aligned}
\mathcal{W}^{s}=\left\{f \in \mathbb{L}^{2}(\mathbb{R}), f \text { admits derivatives up to order } s\right. \\
\text { such that } \left.\|\mid f\|_{s, s o b}^{2}=\|f\|^{2}+\left\|f^{\prime}\right\|^{2}+\ldots+\left\|f^{(s)}\right\|^{2}<+\infty\right\} .
\end{aligned}
$$

Therefore, for $s$ integer, $W_{H}^{s} \subset \mathcal{W}^{s}$. Morevover, the following properties are proved in Bongioanni and Torrea (2006): for all $s>0$,

- $W_{H}^{s} \varsubsetneqq \mathcal{W}^{s}$. If $f \in \mathcal{W}^{s}$ has compact support, then $f \in W_{H}^{s}$.

- $f \in W_{H}^{s} \Rightarrow x^{s} f \in \mathbb{L}^{2}(\mathbb{R})$.

\section{APPEndix B. TAlagRand's inequality}

We recall the Talagrand concentration inequality given in Klein and Rio (2005).

Theorem B.1. Consider $n \in \mathbb{N}^{*}, \mathcal{F}$ a class at most countable of measurable functions, and $\left(X_{i}\right)_{i \in\{1, \ldots, n\}}$ a family of real independent random variables. Define, for $f \in \mathcal{F}, \nu_{n}(f)=$ $(1 / n) \sum_{i=1}^{n}\left(f\left(X_{i}\right)-\mathbb{E}\left[f\left(X_{i}\right)\right]\right)$, and assume that there are three positive constants $M, H$ and $v$ such that $\sup _{f \in \mathcal{F}}\|f\|_{\infty} \leq M, \mathbb{E}\left[\sup _{f \in \mathcal{F}}\left|\nu_{n}(f)\right|\right] \leq H$, and $\sup _{f \in \mathcal{F}}(1 / n) \sum_{i=1}^{n} \operatorname{Var}\left(f\left(X_{i}\right)\right) \leq v$. Then for all $\alpha>0$,

$$
\mathbb{E}\left[\left(\sup _{f \in \mathcal{F}}\left|\nu_{n}(f)\right|^{2}-2(1+2 \alpha) H^{2}\right)_{+}\right] \leq \frac{4}{b}\left(\frac{v}{n} e^{-b \alpha \frac{n H^{2}}{v}}+\frac{49 M^{2}}{b C^{2}(\alpha) n^{2}} e^{-\frac{\sqrt{2} b C(\alpha) \sqrt{\alpha}}{7} \frac{n H}{M}}\right)
$$

with $C(\alpha)=(\sqrt{1+\alpha}-1) \wedge 1$, and $b=\frac{1}{6}$.

By density arguments, this result can be extended to the case where $\mathcal{F}$ is a unit ball of a linear normed space, after checking that $f \rightarrow \nu_{n}(f)$ is continuous and $\mathcal{F}$ contains a countable dense family. 